\title{
Oblique frictional impact of a bar Analysis and comparison of different impact laws
}

\section{Journal Article}

\section{Author(s):}

Payr, Matthias; Glocker, Christoph

Publication date:

2005-09

Permanent link:

https://doi.org/10.3929/ethz-b-000031956

\section{Rights / license:}

In Copyright - Non-Commercial Use Permitted

\section{Originally published in:}

Nonlinear Dynamics 41(4), https://doi.org/10.1007/s11071-005-8200-z 


\title{
Oblique Frictional Impact of a Bar: Analysis and Comparison of Different Impact Laws
}

\author{
MATTHIAS PAYR and CHRISTOPH GLOCKER* \\ IMES - Center of Mechanics, ETH Zurich, 8092 Zurich, Switzerland; \\ *Author for correspondence (e-mail: matthias.payr@imes.mavt.ethz.ch; fax: +41-1-632-1145)
}

(Received: 3 March 2004; accepted: 14 December 2004)

\begin{abstract}
In this paper a basic, easily to multi-contact problems extendable, non-smooth approach is applied to analyze a bar striking an inelastic half-space. Coulomb contact is assumed and modeled by using set-valued Newtonian impact laws in normal as well as in tangential direction. The resulting linear complementarity problem contains all possible impact states and provides an instantaneous collision operator that respects all inequality constraints. This operator depends on the orientation of the bar and determines uniquely the post-impact velocities as functions of the pre-impact state. Different types of solutions may occur, including "stick" and "slip". In this context, stick and slip have to be understood as the two cases characterized by the tangential impulsive force as an element of either the set-valued or of the single-valued domain of the friction law. Depending on the choice of parameters, sign reversal of the tangential contact velocity is possible. For certain inertia properties and initial conditions, the collision operator yields an impact, even for initially vanishing normal contact velocity. This phenomenon is well known as the Painlevé paradox. The results obtained by this fully non-smooth rigid body approach are compared with those of other impact models, such as a lumped mass model with compliance elements, and a collision operator used for particle interactions in flows.
\end{abstract}

Key words: collision operator, complementarity, friction, impact, inequality, lumped mass model

AMR: 160A, 160B, 160Y, 292B, 292Y

\section{Introduction}

During the last years, the concepts used in non-smooth dynamics [1] have been enhanced considerably and successfully applied to many practical examples. The essence of this approach consists for dynamic evolution problems in the structured use of inequalities [2], together with an extended notion of the accelerations in the sense of measure theory [3] that allows for discontinuities in the velocities. One - but not the only one - important field of application of non-smooth dynamics is the rigid body systems theory [4], in which frictional motion and unilateral constraints are formulated and evaluated in a self-contained theoretical framework, which is indispensable in particular for spatial multi-contact situations. Impact-free motion as well as the impacts themselves, complemented by consistent impact laws, are embedded in one unifying description, see e.g. [5] for an overview.

However, there is still too less awareness on how the methods in non-smooth dynamics have to be related to the conventional approaches, in particular with respect to the modelling and the evaluation of impacts. The paper at hand is intended to fill in this gap. The material in [2] contains the general structure of the measure differential inclusions of non-smooth dynamics together with their decomposition into the atomic and the Lebesgue part, which correspond to impacts and impact-free motion. Most of this material, however, concentrates on impact-free motion. Collision laws have not been treated. We, therefore, take only the impact equations from [2], but complement them now by the most basic collision 


\section{M. Payr and C. Glocker}

law, i.e. Newton's frictional impact law in inequality form, to perform a thorough discussion on how they are linked for single collisions to classical approaches.

Conventional methods, which solve potential inequalities most often in an ad hoc manner but do not incorporate them as a substantial part in the collision laws, are mostly applied to the impact at a single contact point of two separate bodies [6]. In such a case, it is quite obvious that a compressive impulsive force in the normal direction is required to prevent bodies from interpenetration and to cause the desired velocity jump. There is no need to worry on how to exclude non-compressive forces from the impact, or how an impact behaves when the impulsive normal force even vanishes. For a single collision, all those cases are of minor interest because one can pre-assume a positive value of the normal impulsive force. Such an ad hoc approach works even for planar frictional collisions. The different cases like gross slip, slip with sign reversal and stick, may be checked for validity and evaluated in a very straightforward manner, as soon as the impact law has been formulated. Such approaches may be found in [7-9], just to mention some out of a huge number of contributions [5].

Things, however, become much more complicated as soon as more than one unilateral contact is closed at the time of a collision. Such an event may be called an impact within a multi-contact situation. Closed contacts may - or may not - react by compressive impulsive forces on a collision at a different location in the system. They may start to open or perform induced stick-slip transitions. Even worse, the problem of simultaneous collisions of different contacts has to be attacked. This is necessary in view of numerical schemes that approximate the evolution of a collision system by taking finite steps in time. In particular, the fact that one is able to handle a single collision of two separate free bodies by both analytical and numerical methods does not yet ensure that one is also able to perform efficient numerical simulation of the general case. At least two steps are necessary to move from an intuitive evaluation of a single collision to the general multi-contact situation: First of all, a complete and unambiguous description of the constitutive equations of the impact has to be provided, containing all the possible states of the impact as well as the associated transition rules and all inequality restrictions on the impulsive forces and relative velocities. The most consistent and promising way is to formulate them in terms of inclusions by using set-valued maximal maps as done in Sections 2 and 3. Secondly, one has to look for numerical methods that allow for an efficient treatment of the inequalities in the impact constitutive equations. There is no way to evaluate the inequalities ad hoc by trial and error methods, because the complexity of the system raises with the power of contacts involved. One - but not the only one - approach is to formulate the system as a linear complementarity problem and to invoke an appropriate solver.

For a comparative study of both approaches, it is therefore necessary to narrow down the general impact operator from non-smooth dynamics to the planar case of a single impacting contact between two bodies. As a representative example and without loss of generality, one can further choose the impact configuration of a rigid bar striking an inelastic half-space, which is considered as one of the benchmark problems in impact dynamics. This example, general enough to still contain Painlevé's problem, is analyzed in full detail with respect to the standard impact law in non-smooth dynamics. In contrast to [4], in which some numerical results on the frictional sliding motion of the bar and Poisson's impact law have been presented, this paper focusses on the frictional collision and analyzes all possible impact states when Newton's impact law is used.

The purpose of this paper is thus twofold: Firstly, we want to present in a most condensed and concise manner the idea of the non-smooth inequality approach to impacts and to perform all necessary steps of evaluation by the example of the striking rigid bar. Secondly, we want to relate these results to other methods found in literature. In particular, we have chosen Walton's instantaneous collision operator for rigid spheres which is used in [8] to conduct numerical simulations on particle-loaded flows. This 
collision operator provides the link to compare the results of the non-smooth inequality approach even with Stronge's lumped mass model [7]. Stronge treats the frictional impact by introducing compliant contact elements. In his model, an energetic impact coefficient $\varepsilon_{*}$ is defined such that the work done in the normal direction is reversible for $\varepsilon_{*}=1$, even if friction in tangential direction is present.

The paper is organized as follows: In Section 2 we introduce the two most basic set-valued maps defined on $\mathbb{R}$, the unilateral primitive $\operatorname{Upr}(x)$ and the filled-in relay function $\operatorname{Sgn}(x)$. It is shown that the relay function can be decomposed into two unilateral primitives, which will turn out to be quintessential when the linear complementarity problem of the impacting bar is formulated.

The theoretical framework on how to state a frictional impact problem in finite freedom dynamics is shortly presented in Section 3. We follow the work in [1], but narrow it already down to planar unilateral Coulomb contacts with Newtonian impacts in order to have access to linear complementarity theory later on. We give a complete formulation of the dynamic impact process based on an inclusion involving the difference in the post- and pre-impact velocities, and try to clearly arrange the complete set of frictional contact-impact laws.

In Section 4, this approach is applied to the example of a rigid bar striking an inelastic half-space by a frictional impact. The impact equations and impact laws are formulated for this event in terms of contact velocities and contact impulses, from which a linear complementarity problem of dimension 3 in standard form is obtained. Three parameters are used to characterize the impact, which are the normal and tangential restitution coefficients $\varepsilon_{\mathrm{N}}$ and $\varepsilon_{\mathrm{T}}$, and the friction coefficient $\mu$.

Section 5 deals with the complete analytical evaluation of the impact linear complementarity problem from Section 4. All $2^{3}=8$ different cases of this problem are checked combinatorially for solutions. It turns out that only three of them correspond to collisions, called "backward slip", "forward slip" and "stick". These three impact states are further investigated for existence, depending on the angle at which the bar strikes the half-space, and analyzed with respect to the occurrence of sign reversal of the tangential relative velocity. A critical maximal coefficient of friction is derived, assuring all three impact states to be accessible.

For supercritical friction, the existence of impacts without collisions is shown in Section 6 by considering situations at which the tip of the bar approaches the surface nearly tangentially. For the limit of a completely flat approaching process, pre-impact sliding motion is obtained which connects these results to the Painlevé paradox, cf. Painlevé [10], Génot and Brogliato [11], and Leine et al. [12].

In Section 7, the presented non-smooth inequality complementarity approach is finally compared with different other frictional impact models that are found in literature. In particular, the collision operator for a sphere is derived from the impact geometry of the striking bar to show equivalence with Walton's approach [8]. This serves as the link to Stronge's energetic restitution coefficient, which is used together with a lumped mass model [7]. Final conclusions summarizing the obtained results are given in Section 8.

\section{Basic Set-Valued Elements}

A linear complementarity problem (LCP) is a problem of the following form [13, 14]: For given $\mathbf{A} \in \mathbb{R}^{n, n}$ and $\mathbf{b} \in \mathbb{R}^{n}$, find $\mathbf{x} \in \mathbb{R}^{n}$ and $\mathbf{y} \in \mathbb{R}^{n}$ such that the linear equation $\mathbf{y}=\mathbf{A x}+\mathbf{b}$ holds together with the complementarity conditions $y_{i} \geq 0, x_{i} \geq 0, y_{i} x_{i}=0$ for $i=1, \ldots, n$. The latter conditions are often written in the form $\mathbf{y} \succeq 0, \mathbf{x} \succeq 0, \mathbf{y}^{\top} \mathbf{x}=0$ or, equivalently, as $0 \preceq \mathbf{y} \perp \mathbf{x} \succeq 0$.

It is convenient to introduce a maximal monotone set-valued map defined on $\mathbb{R}^{+}$, which has been named in [15] the unilateral primitive Upr, and which is the most important multifunction related to 

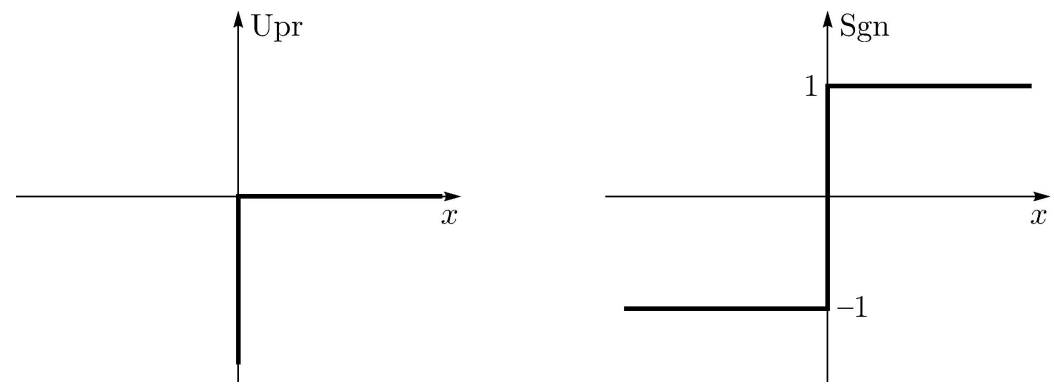

Figure 1. The maps $x \rightarrow \operatorname{Upr}(x)$ and $x \rightarrow \operatorname{Sgn}(x)$.

complementarity,

$$
\operatorname{Upr}(x):= \begin{cases}\{0\} & \text { if } x>0 \\ (-\infty, 0] & \text { if } x=0\end{cases}
$$

The graph of this map is depicted in the left part of Figure 1. Apparently, we are now able to express each complementarity condition of the LCP by one inclusion, since

$$
-y \in \operatorname{Upr}(x) \Leftrightarrow y \geq 0, \quad x \geq 0, \quad x y=0 .
$$

Unilateral primitives are used in mechanics on displacement and on velocity level to model unilateral geometric and kinematic constraints, such as free plays with stops, sprag clutches and the like. The associated set-valued force laws are conveniently stated as inclusions in the form (2).

A second maximal monotone set-valued map frequently met in non-smooth systems is the filled-in relay function $\operatorname{Sgn}(x)$, defined by

$$
\operatorname{Sgn}(x):= \begin{cases}\{+1\} & \text { if } x>0 \\ {[-1,+1]} & \text { if } x=0 \\ \{-1\} & \text { if } x<0\end{cases}
$$

see Figure 1 for the graph. Note the difference at $x=0$ to the classical sgn-function, which is defined as $\operatorname{sgn}(x=0)=0$. In mechanics, relay functions on velocity level are used to model any kind of dry friction. On displacement level, they describe the behavior of pre-stressed springs. The relay function (3) can be represented by two unilateral primitives as indicated in Figure 2, which yields in terms of inclusions

$$
-y \in \operatorname{Sgn}(x) \Leftrightarrow \exists x_{\mathrm{R}}, x_{\mathrm{L}} \text { such that }\left\{\begin{array}{l}
-y \in+\mathrm{Upr}\left(x_{\mathrm{R}}\right)+1 \\
-y \in-\operatorname{Upr}\left(x_{\mathrm{L}}\right)-1 . \\
x=x_{\mathrm{R}}-x_{\mathrm{L}}
\end{array}\right.
$$

More details on this decomposition can be found in [2] together with various applications of even more complex set-valued interaction laws and their representations via unilateral primitives and inclusions. 

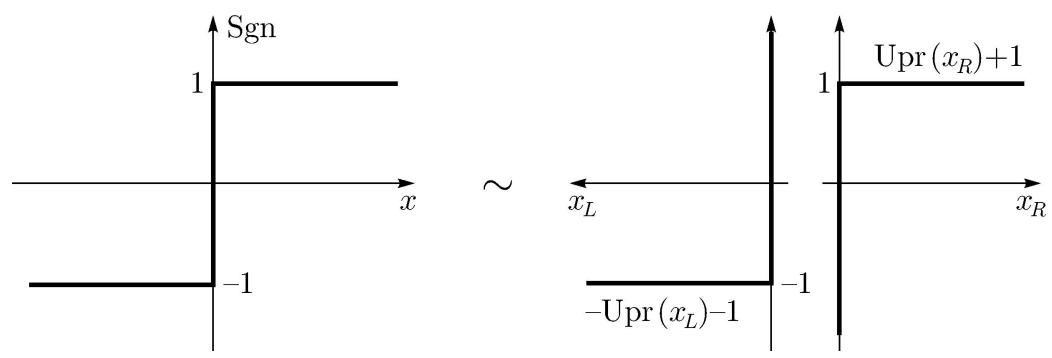

Figure 2. Decomposition of Sgn into Upr.

By using (2), we may finally express (4) in terms of complementarities,

$$
-y \in \operatorname{Sgn}(x) \Leftrightarrow \exists x_{\mathrm{R}}, x_{\mathrm{L}} \text { such that }\left\{\begin{array}{l}
1+y \geq 0, x_{\mathrm{R}} \geq 0,(1+y) x_{\mathrm{R}}=0 \\
1-y \geq 0, x_{\mathrm{L}} \geq 0,(1-y) x_{\mathrm{L}}=0 . \\
x=x_{\mathrm{R}}-x_{\mathrm{L}}
\end{array}\right.
$$

This representation has to be used when a problem involving Sgn-multifunctions is formulated as an LCP in standard form.

Both, the unilateral primitive as well as the relay function, may be expressed in terms of the subdifferential of certain convex functions. In particular, $\operatorname{Sgn}(x)=\partial|x|$ and $\operatorname{Upr}(x)=\partial \boldsymbol{I}_{\mathbb{R}^{+}}(x)$, where $I_{\mathbb{R}^{+}}$denotes the indicator function of the set of non-negative real numbers. The important fact that the subdifferential of lower semi-continuous proper convex functions provides maximal monotone set-valued maps, links differential inclusions to variational analysis and optimization theory.

\section{Planar Frictional Contacts in Rigid Body Systems}

In this section, we briefly review the full non-smooth rigid body approach to impacts. We state the complete set of equations and inclusions that are necessary to solve a frictional impact event in an autonomous multibody multi-contact system, based on a Newton-type inequality restitution law combined with Coulomb friction. Precisely this approach is applied to the oblique frictional impact of a rigid bar in the next section. For a detailed description of the material presented here, we refer in particular to [1, $3]$, to [2, 4] and to the concise paper [15], in which adjoined impact-free motion is theoretically taken into account and numerically evaluated together with the impacts by a time-stepping scheme.

We consider an $f$-dimensional mechanical system with frictional unilateral contacts. The set of generalized coordinates is denoted by $\mathbf{q} \in \mathbb{R}^{f}$, and the associated velocities by $\mathbf{u} \in \mathbb{R}^{f}$ with $\dot{\mathbf{q}}=\mathbf{u}$. We further denote by $\mathbf{u}^{+}(t)$ and $\mathbf{u}^{-}(t)$ the generalized post- and pre-impact velocities, respectively, which might be different from each other in the case of an impact. For such a system, the Newton-Euler equations in integrated form, called the impact equations, read as

$$
\mathbf{M}\left(\mathbf{u}^{+}-\mathbf{u}^{-}\right)=\mathbf{R}
$$

where $\mathbf{M}(\mathbf{q})$ is the symmetric and positive definite mass matrix of the system, and $\mathbf{R}$ contains the impulsive contact forces. Finite forces, such as gravity or reactions from springs or dashpots, do not contribute to the integral and are thus not to be considered in (6). 
We assume a total of $n$ frictional unilateral constraints in the system, which are represented by $n$ inequalities

$$
g_{\mathrm{N} i}(\mathbf{q}) \geq 0, \quad i=1, \ldots, n
$$

The $g_{\mathrm{N} i}$ are the gap functions of the frictional contacts. They are formulated such that $g_{\mathrm{N} i}>0$ indicates an open contact with an Euclidean distance of the contact points given by the value of $g_{\mathrm{N} i} ; g_{\mathrm{N} i}=0$ corresponds to a closed contact, and $g_{\mathrm{N} i}<0$ indicates forbidden overlapping or interpenetration. A detailed description on how to define these inequalities in a multibody system may be found in $[2,4]$. We further introduce the set of active contacts

$$
\mathcal{H}(t)=\left\{i \mid g_{\mathrm{N} i}(\mathbf{q}(t))=0\right\}
$$

which singles out the contacts at which contact forces may occur. The generalized impulsive forces $\mathbf{R}$ in (6) are therefore at most composed of the normal and tangential contact forces of the individuals $i \in \mathcal{H}$ and may be written as

$$
\mathbf{R}=\sum_{i \in \mathcal{H}} \mathbf{w}_{\mathrm{N} i} \Lambda_{\mathrm{N} i}+\mathbf{w}_{\mathrm{T} i} \Lambda_{\mathrm{T} i}
$$

In this expression, $\mathbf{w}_{\mathrm{N} i}(\mathbf{q})$ and $\mathbf{w}_{\mathrm{T} i}(\mathbf{q})$ are the generalized normal and tangential force directions, and $\Lambda_{\mathrm{N} i}$ and $\Lambda_{\mathrm{T} i}$ the associated scalar normal and tangential contact impulses of contact $i$.

The system's dynamics is not yet completely determined by Equations (6)-(9) because we still have to specify impact laws that relate the contact impulsive forces $\Lambda_{\mathrm{N} i}$ and $\Lambda_{\mathrm{T} i}$ to the system's kinematic state $(\mathbf{q}, \mathbf{u})$. To do so, we introduce first the normal and tangential relative velocities in the contacts,

$$
\gamma_{\mathrm{N} i}=\mathbf{w}_{\mathrm{N} i}^{\top} \mathbf{u}, \quad \gamma_{\mathrm{T} i}=\mathbf{w}_{\mathrm{T} i}^{\top} \mathbf{u}
$$

with $\mathbf{w}_{\mathrm{N} i}(\mathbf{q})$ and $\mathbf{w}_{\mathrm{T} i}(\mathbf{q})$ as in (9), see e.g. [4] on how to obtain these terms. We choose for the normal direction of each contact a unilateral version of Newton's impact law with local restitution coefficient $\varepsilon_{\mathrm{N} i} \in[0,1]$, and for the tangential direction a Coulomb-type frictional law with friction coefficient $\mu_{i}$ that is complemented by tangential restitution behavior $\varepsilon_{\mathrm{T} i} \in[0,1]$. We define

$$
\xi_{\mathrm{N} i}:=\gamma_{\mathrm{N} i}^{+}+\varepsilon_{\mathrm{N} i} \gamma_{\mathrm{N} i}^{-}, \quad \xi_{\mathrm{T} i}:=\gamma_{\mathrm{T} i}^{+}+\varepsilon_{\mathrm{T} i} \gamma_{\mathrm{T} i}^{-}
$$

where $\left(\gamma_{\mathrm{N} i}^{ \pm}, \gamma_{\mathrm{T} i}^{ \pm}\right):=\left(\gamma_{\mathrm{N} i}, \gamma_{\mathrm{T} i}\right)\left(\mathbf{u}^{ \pm}\right)$, and pose the normal and tangential impact laws as

$$
-\Lambda_{\mathrm{N} i} \in \operatorname{Upr}\left(\xi_{\mathrm{N} i}\right), \quad-\Lambda_{\mathrm{T} i} \in \mu_{i} \Lambda_{\mathrm{N} i} \operatorname{Sgn}\left(\xi_{\mathrm{T} i}\right)
$$

With Equations (6)-(12) we have now obtained a complete description of the impact dynamics of the system which will be applied in the next section to a single frictional oblique impact of a rigid bar. A general method on how to rewrite (6)-(12) as a linear complementarity problem in standard form is presented in [15]. 
Energetic consistency of the scleronomic multi-contact impact problem is guaranteed if the restitution coefficients of the active contacts are all equal to each other, $\varepsilon:=\varepsilon_{\mathrm{N} i}=\varepsilon_{\mathrm{T} j} \forall i, j \in \mathcal{H}(t)$. The associated proof can be found in [16] and is based on the following strategy. The difference in kinetic energy,

$$
T^{+}-T^{-}=\frac{1}{2} \mathbf{u}^{+\top} \mathbf{M} \mathbf{u}^{+}-\frac{1}{2} \mathbf{u}^{-\top} \mathbf{M} \mathbf{u}^{-},
$$

is reformulated in terms of the local contact variables, i.e. in terms of those variables $\left(\Lambda_{\mathrm{NT} i}, \xi_{\mathrm{NT} i}\right)$ that have been used to state the constitutive equations (12). By using (6), (9)-(11), this results in an expression

$$
T^{+}-T^{-}=\frac{1}{1+\varepsilon} \boldsymbol{\Lambda}^{\top} \boldsymbol{\xi}-\frac{1}{2} \frac{1-\varepsilon}{1+\varepsilon} \boldsymbol{\Lambda}^{\top}\left(\mathbf{W}^{\top} \mathbf{M}^{-1} \mathbf{W}\right) \boldsymbol{\Lambda}
$$

with the matrix and vector quantities being composed of both the normal and tangential contributions for all $i \in \mathcal{H}$, i.e. $\mathbf{W}=\left(\mathbf{w}_{\mathrm{N} i}, \mathbf{w}_{\mathrm{T} i}\right), \boldsymbol{\Lambda}=\left(\Lambda_{\mathrm{N} i}, \Lambda_{\mathrm{T} i}\right)^{\top}, \boldsymbol{\xi}=\left(\xi_{\mathrm{N} i}, \xi_{\mathrm{T} i}\right)^{\top}$. By the common restriction of the restitution coefficient $\varepsilon$ to values $\varepsilon \in[0,1]$, one immediately recognizes that $1 / 2 \frac{1-\varepsilon}{1+\varepsilon} \boldsymbol{\Lambda}^{\top}\left(\mathbf{W}^{\top} \mathbf{M}^{-1} \mathbf{W}\right) \boldsymbol{\Lambda} \geq 0$ because $\mathbf{W}^{\top} \mathbf{M}^{-1} \mathbf{W}$ is (at least) positive semi-definite. For the first term on the right-hand side in (14), it holds that

$$
\boldsymbol{\Lambda}^{\top} \boldsymbol{\xi}=\sum_{i \in \mathcal{H}}\left(\Lambda_{\mathrm{N} i} \xi_{\mathrm{N} i}+\Lambda_{\mathrm{T} i} \xi_{\mathrm{T} i}\right) \leq 0 .
$$

This is a direct consequence on the constitutive equations (12) for which $\Lambda_{\mathrm{N} i} \xi_{\mathrm{N} i}=0$ by the complementarity (2), and $\Lambda_{\mathrm{T} i} \xi_{\mathrm{T} i} \leq 0$ due to the relay function and the restriction $\Lambda_{\mathrm{N} i} \geq 0$. Kinetic energy is preserved by the impact if, for example, $\varepsilon=1$ and $\mu_{i} \gg 1$ has been chosen. For this case, the last term in (14) drops out and one has, in addition, $\Lambda_{\mathrm{T} i} \xi_{\mathrm{T} i}=0$. For the contacts participating in the impact by non-vanishing impulsive normal forces $\Lambda_{\mathrm{N} i}>0$, the relative velocities in both the normal and tangential directions are inverted according to $\xi_{\mathrm{N} i}=\xi_{\mathrm{T} i}=0$ from (12), which gives $\gamma_{\mathrm{N} i}^{+}=-\gamma_{\mathrm{N} i}^{-}$ and $\gamma_{\mathrm{T} i}^{+}=-\gamma_{\mathrm{T} i}^{-}$by $(11)$.

\section{Frictional Impact of a Bar Against a Rigid Half-Space}

In the following, we study the dynamics of a rigid bar (mass $m$, barycentric moment of inertia $\Theta_{\mathrm{S}}$, length $2 s$, center of gravity $S$ ) striking an inelastic half-space (Figure 3). Our aim is to determine by Equations (6)-(12) the post-impact velocities of the bar and the impulsive forces acting at the contact point C. According to the framework shown in Section 3, Coulomb type of friction with a coefficient of friction $\mu>0$ is assumed. Normal and tangential restitution is taken into account by the coefficients
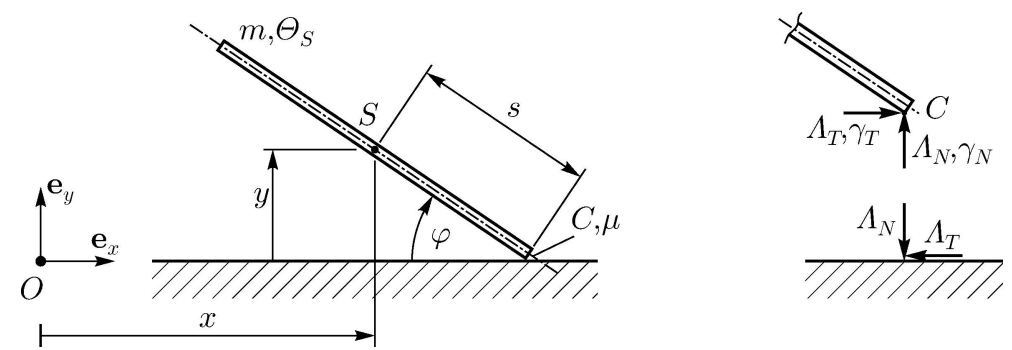

Figure 3. Left: Mechanical model of a rigid bar striking an inelastic half-space. Right: Free body diagram showing the impulsive forces $\Lambda_{\mathrm{N}}, \Lambda_{\mathrm{T}}$ and the relative velocities $\gamma_{\mathrm{N}}, \gamma_{\mathrm{T}}$ at contact point $\mathrm{C}$. 
$\varepsilon_{\mathrm{N}} \in[0,1]$ and $\varepsilon_{\mathrm{T}} \in[0,1]$, respectively. The evaluation of the impact behavior is done in the framework of linear complementarity. The associated linear complementarity problem of dimension 3 is derived in this section and solved analytically in Section 5.

The position of the bar with respect to the inertial frame $\left(\mathrm{O}, \mathbf{e}_{x}, \mathbf{e}_{y}\right)$ is described by the absolute linear displacements $x, y$ of its center of mass $S$, and the absolute angular displacement $\varphi$ as shown in the left part of Figure 3. We, thus, define the vector of generalized coordinates to be $\mathbf{q}:=(x, y, \varphi)^{\top}$ with associated velocities $\dot{\mathbf{q}}=\mathbf{u}:=\left(u_{x}, u_{y}, u_{\varphi}\right)^{\top}$, i.e. $\dot{x}=u_{x}, \dot{y}=u_{y}, \dot{\varphi}=u_{\varphi}$. The gap $g_{\mathrm{N}}(\mathbf{q})$ between the tip $\mathrm{C}$ of the bar and the environment results directly from the displacement $\mathbf{r}_{\mathrm{OC}}$ of $\mathrm{C}$ relative to $\mathrm{O}$,

$$
\mathbf{r}_{\mathrm{OC}}=\left(\begin{array}{c}
x+s \cos \varphi \\
y-s \sin \varphi
\end{array}\right) \Rightarrow g_{\mathrm{N}}(\mathbf{q})=y-s \sin \varphi \geq 0
$$

and sets up a unilateral constraint $g_{\mathrm{N}}(\mathbf{q}) \geq 0$ due to impenetrability. Note that $g_{\mathrm{N}}=0 \Leftrightarrow y=s \sin \varphi$ for a closed contact that we meet at the instance of a collision. We are interested in single contacts only. Thus, a simultaneous contact of the rod's second tip is excluded from the succeeding discussions, i.e. we restrict ourselves to $0<\varphi<\pi$ whenever contact $\mathrm{C}$ is closed by $g_{\mathrm{N}}=0$ or, equivalently, $y=s \sin \varphi$. As a consequence, the set $\mathcal{H}$ in (8) consists of one element only, which simplifies (9)-(12) in the sense that summation is not needed and that the lower index $i$ to distinguish between the different active contacts can be dropped.

By differentiation of $\mathbf{r}_{\mathrm{OC}}$ in (16) with respect to time, one obtains the velocities of the rod's tip C relative to the non-moving surface of the environment. In this case, they already constitute the contact relative velocities $\gamma_{\mathrm{N}}$ in the normal and $\gamma_{\mathrm{T}}$ in the tangential directions,

$$
\dot{\mathbf{r}}_{\mathrm{OC}}=\left(\begin{array}{c}
u_{x}-s u_{\varphi} \sin \varphi \\
u_{y}-s u_{\varphi} \cos \varphi
\end{array}\right)=\left(\begin{array}{c}
\gamma_{\mathrm{T}}(\mathbf{u}) \\
\gamma_{\mathrm{N}}(\mathbf{u})
\end{array}\right) \text {. }
$$

According to (10) and due to our particular choice of $\mathbf{u}=\left(u_{x}, u_{y}, u_{\varphi}\right)^{\top}$, one is able to identify from this equation the terms $\mathbf{w}_{\mathrm{N}}$ and $\mathbf{w}_{\mathrm{T}}$ as displayed in (19).

Let $t=0$ be the time of impact at which the gap function closes $\left(g_{\mathrm{N}}=0\right)$ with a negative normal contact velocity $\gamma_{\mathrm{N}}^{-} \equiv \dot{g}_{\mathrm{N}}^{-}<0$. As introduced in Section 3, pre-impact variables $(t \uparrow 0)$ are characterized by the superscript ${ }^{-}$, and post-impact variables $(t \downarrow 0)$ by the superscript ${ }^{+}$. The dynamics at the impact has to follow the Newton-Euler equations in integrated form,

$$
\begin{aligned}
m\left(u_{x}^{+}-u_{x}^{-}\right) & =\Lambda_{\mathrm{T}}, \\
m\left(u_{y}^{+}-u_{y}^{-}\right) & =\Lambda_{\mathrm{N}}, \\
\Theta_{\mathrm{S}}\left(u_{\varphi}^{+}-u_{\varphi}^{-}\right) & =-\Lambda_{\mathrm{N}} s \cos \varphi-\Lambda_{\mathrm{T}} s \sin \varphi,
\end{aligned}
$$

with $\Lambda_{\mathrm{N}}$ the normal and $\Lambda_{\mathrm{T}}$ the tangential impulsive contact force according to the free body diagram in Figure 3. In order to rewrite (18) in standard form (6) and (9), the mass matrix $\mathbf{M}$ and the generalized force directions $\mathbf{w}_{\mathrm{N}}, \mathbf{w}_{\mathrm{T}}$ have to be identified. This yields

$$
\mathbf{M}=\left(\begin{array}{ccc}
m & 0 & 0 \\
0 & m & 0 \\
0 & 0 & \Theta_{\mathrm{S}}
\end{array}\right), \quad \mathbf{w}_{\mathrm{N}}=\left(\begin{array}{c}
0 \\
1 \\
-s \cos \varphi
\end{array}\right), \quad \mathbf{w}_{\mathrm{T}}=\left(\begin{array}{c}
1 \\
0 \\
-s \sin \varphi
\end{array}\right)
$$

with the same vectors $\mathbf{w}_{\mathrm{N}}, \mathbf{w}_{\mathrm{T}}$ as already found in the relative velocities (17). 
Thanks to (19), the impact Equations (6) and (9) and relative kinematics (10) are now fully determined and available in standard form,

$$
\begin{aligned}
& \mathbf{M}\left(\mathbf{u}^{+}-\mathbf{u}^{-}\right)=\mathbf{w}_{\mathrm{N}} \Lambda_{\mathrm{N}}+\mathbf{w}_{\mathrm{T}} \Lambda_{\mathrm{T}}, \\
& \gamma_{\mathrm{N}}=\mathbf{w}_{\mathrm{N}}^{\top} \mathbf{u}, \quad \gamma_{\mathrm{T}}=\mathbf{w}_{\mathrm{T}}^{\top} \mathbf{u} .
\end{aligned}
$$

Problem (20) can be rewritten in terms of solely contact variables. To do so, we first state the differences in the pre- and post-impact relative velocities and eliminate in a second step the generalized velocities $\mathbf{u}^{ \pm}$with the help of the first Equation in (20),

$$
\begin{aligned}
& \gamma_{\mathrm{N}}^{+}-\gamma_{\mathrm{N}}^{-}=\mathbf{w}_{\mathrm{N}}^{\top}\left(\mathbf{u}^{+}-\mathbf{u}^{-}\right)=\mathbf{w}_{\mathrm{N}}^{\top} \mathbf{M}^{-1}\left(\mathbf{w}_{\mathrm{N}} \Lambda_{\mathrm{N}}+\mathbf{w}_{\mathrm{T}} \Lambda_{\mathrm{T}}\right), \\
& \gamma_{\mathrm{T}}^{+}-\gamma_{\mathrm{T}}^{-}=\mathbf{w}_{\mathrm{T}}^{\top}\left(\mathbf{u}^{+}-\mathbf{u}^{-}\right)=\mathbf{w}_{\mathrm{T}}^{\top} \mathbf{M}^{-1}\left(\mathbf{w}_{\mathrm{N}} \Lambda_{\mathrm{N}}+\mathbf{w}_{\mathrm{T}} \Lambda_{\mathrm{T}}\right) .
\end{aligned}
$$

By introducing the quantities

$$
\begin{aligned}
& \alpha=\alpha(\varphi):=\mathbf{w}_{\mathrm{N}}^{\top} \mathbf{M}^{-1} \mathbf{w}_{\mathrm{N}}=m^{-1}+\Theta_{\mathrm{S}}^{-1} s^{2} \cos ^{2} \varphi>0, \\
& \beta=\beta(\varphi):=\mathbf{w}_{\mathrm{T}}^{\top} \mathbf{M}^{-1} \mathbf{w}_{\mathrm{T}}=m^{-1}+\Theta_{\mathrm{S}}^{-1} s^{2} \sin ^{2} \varphi>0, \\
& \delta=\delta(\varphi):=\mathbf{w}_{\mathrm{N}}^{\top} \mathbf{M}^{-1} \mathbf{w}_{\mathrm{T}}=\mathbf{w}_{\mathrm{T}}=\mathbf{w}_{\mathrm{T}}^{\top} \mathbf{M}^{-1} \mathbf{w}_{\mathrm{N}}=\Theta_{\mathrm{S}}^{-1} s^{2} \sin \varphi \cos \varphi,
\end{aligned}
$$

Equations in (21) may now be written in the form

$$
\gamma_{\mathrm{N}}^{+}-\gamma_{\mathrm{N}}^{-}=\alpha \Lambda_{\mathrm{N}}+\delta \Lambda_{\mathrm{T}}, \quad \gamma_{\mathrm{T}}^{+}-\gamma_{\mathrm{T}}^{-}=\delta \Lambda_{\mathrm{N}}+\beta \Lambda_{\mathrm{T}}
$$

Only for $\delta=0$, e.g. for a sphere $(\varphi \equiv \pi / 2)$ or a bar with an orientation of $\varphi \in\{0, \pi / 2, \pi\}$, these two equations become decoupled. It is then possible to solve the normal impact subproblem separately and use the results for the tangential impact, for which the normal impulsive force $\Lambda_{\mathrm{N}}$ is needed in the tangential impact law (12). All other configurations result in coupled normal and tangential equations.

In the following, an LCP formulation of the frictional impact problem is derived. Equations (23) do not yet carry the whole information needed but have to be completed by the terms from (11)

$$
\xi_{\mathrm{N}}:=\gamma_{\mathrm{N}}^{+}+\varepsilon_{\mathrm{N}} \gamma_{\mathrm{N}}^{-}, \quad \xi_{\mathrm{T}}:=\gamma_{\mathrm{T}}^{+}+\varepsilon_{\mathrm{T}} \gamma_{\mathrm{T}}^{-},
$$

and the normal and tangential set-valued impact laws (12),

$$
-\Lambda_{\mathrm{N}} \in \operatorname{Upr}\left(\xi_{\mathrm{N}}\right), \quad-\Lambda_{\mathrm{T}} \in \mu \Lambda_{\mathrm{N}} \operatorname{Sgn}\left(\xi_{\mathrm{T}}\right)
$$

The Sgn-multifunction in the second inclusion in (25) is now decomposed according to (4) into two unilateral primitives,

$$
\begin{cases}-\Lambda_{\mathrm{TR}} \in \operatorname{Upr}\left(\xi_{\mathrm{TR}}\right) & \text { with } \Lambda_{\mathrm{TR}}=\mu \Lambda_{\mathrm{N}}+\Lambda_{\mathrm{T}} \\ -\Lambda_{\mathrm{TL}} \in \operatorname{Upr}\left(\xi_{\mathrm{TL}}\right) & \text { with } \Lambda_{\mathrm{TL}}=\mu \Lambda_{\mathrm{N}}-\Lambda_{\mathrm{T}} \\ \xi_{\mathrm{T}}=\xi_{\mathrm{TR}}-\xi_{\mathrm{TL}} & \end{cases}
$$

in order to have access to complementarity formulations (5). After elimination of $\gamma_{\mathrm{N}}^{+}, \gamma_{\mathrm{T}}^{+}, \xi_{\mathrm{T}}, \Lambda_{\mathrm{T}}$ from (23), (24) and (26), the resulting linear equations are

$$
\left(\begin{array}{c}
\xi_{\mathrm{N}} \\
\xi_{\mathrm{TR}} \\
\Lambda_{\mathrm{TL}}
\end{array}\right)=\left(\begin{array}{ccc}
\alpha-\mu \delta & \delta & 0 \\
\delta-\mu \beta & \beta & 1 \\
2 \mu & -1 & 0
\end{array}\right)\left(\begin{array}{c}
\Lambda_{\mathrm{N}} \\
\Lambda_{\mathrm{TR}} \\
\xi_{\mathrm{TL}}
\end{array}\right)+\left(\begin{array}{c}
\left(1+\varepsilon_{\mathrm{N}}\right) \gamma_{\mathrm{N}}^{-} \\
\left(1+\varepsilon_{\mathrm{T}}\right) \gamma_{\mathrm{T}}^{-} \\
0
\end{array}\right) .
$$


Together with the complementarity conditions

$$
0 \preceq\left(\begin{array}{c}
\xi_{\mathrm{N}} \\
\xi_{\mathrm{TR}} \\
\Lambda_{\mathrm{TL}}
\end{array}\right) \perp\left(\begin{array}{c}
\Lambda_{\mathrm{N}} \\
\Lambda_{\mathrm{TR}} \\
\xi_{\mathrm{TL}}
\end{array}\right) \succeq 0,
$$

they set up a linear complementarity problem in standard form. This formulation, i.e. (27) and (28), contains all the possible post-impact states from the original impact laws (25). A detailed description on how to derive complementarity formulations for the general case of several impacting or closed contacts can be found in [15].

\section{Analysis of the Impact Equations}

The LCP (27) and (28) derived in the previous section comprises all physically possible impact states. In general, all $2^{\mathrm{n}}(n=3)$ cases defined by any possible complementary combination of non-zero variables in (28) have to be checked for a given pre-impact state to find the one which solves (27) and (28) without contradictions and determines the desired post-impact velocities. However, only four of them are needed to classify the frictional impact because the remaining four correspond to non-impulsive behavior: From physical imagination, it is clear that a non-vanishing normal impulse $\Lambda_{\mathrm{N}}>0$ is needed to stop the bar, which approaches the ground with a presumed normal pre-impact relative velocity $\gamma_{\mathrm{N}}^{-}<0$. This is formally proven by contradiction:

Assume that $\gamma_{\mathrm{N}}^{-}<0$, but $\Lambda_{\mathrm{N}}=0$. This yields by the second inclusion in (25) that $\Lambda_{\mathrm{T}}=0$. Thus, $\Lambda_{\mathrm{TR}}=\Lambda_{\mathrm{TL}}=0$ by (26). With this result, the first equation in (27) becomes $\xi_{\mathrm{N}}=\left(1+\varepsilon_{\mathrm{N}}\right) \gamma_{\mathrm{N}}^{-}$, which contradicts $\xi_{\mathrm{N}}>0$ in (28) because $\gamma_{\mathrm{N}}^{-}<0$ was assumed. We, therefore, know that $\Lambda_{\mathrm{N}}>0$, which means by the complementarities (28) that $\xi_{\mathrm{N}}=0$ for the impact. Hence, the normal relative velocity is inverted according to (24) by $\gamma_{\mathrm{N}}^{+}=-\varepsilon_{\mathrm{N}} \gamma_{\mathrm{N}}^{-}$independent of the tangential impact behavior. We further see that only four combinations of complementarities in (27) remain because the other four corresponding to $\left(\xi_{\mathrm{N}}>0, \Lambda_{\mathrm{N}}=0\right)$ are not feasible for our example and have already been excluded.

Three out of the four remaining cases provide a non-vanishing normal impulse $\Lambda_{\mathrm{N}}>0$ and are now discussed in more detail. The only reasonable classification of the frictional impact is based on the direction and absolute value of the tangential impulsive force $\Lambda_{\mathrm{T}}$, corresponding to the three different branches of the Sgn-multifunction in the tangential impact law (25).

- Backward slip: This case is defined by a tangential impulse at the positive bound of the friction interval $\left(\Lambda_{\mathrm{T}}=+\mu \Lambda_{\mathrm{N}}\right)$.

Assume that $\Lambda_{\mathrm{N}}>0$ and $\Lambda_{\mathrm{T}}=+\mu \Lambda_{\mathrm{N}}$. This means by (26) that $\Lambda_{\mathrm{TL}}=0$ and $\Lambda_{\mathrm{TR}}>0$. Thus, $\xi_{\mathrm{N}}=0$ and $\xi_{\mathrm{TR}}=0$, resulting from the complementarities (28). By setting $\xi_{\mathrm{N}}=0, \xi_{\mathrm{TR}}=0, \Lambda_{\mathrm{TL}}=0$ in (27), these linear equations can be solved uniquely for $\Lambda_{\mathrm{N}}, \Lambda_{\mathrm{TR}}, \xi_{\mathrm{TL}}$. Equation (26) is then used to determine $\xi_{\mathrm{T}}$, and finally (24) to compute the post-impact relative velocities $\gamma_{\mathrm{N}}^{+}$and $\gamma_{\mathrm{T}}^{+}$. The results are

$$
\begin{aligned}
& \Lambda_{\mathrm{N}}=\frac{1+\varepsilon_{\mathrm{N}}}{-\alpha-\mu \delta} \gamma_{\mathrm{N}}^{-}, \\
& \Lambda_{\mathrm{T}}=\mu \Lambda_{\mathrm{N}}, \\
& \gamma_{\mathrm{N}}^{+}=-\varepsilon_{\mathrm{N}} \gamma_{\mathrm{N}}^{-}, \\
& \gamma_{\mathrm{T}}^{+}=(\delta+\mu \beta) \Lambda_{\mathrm{N}}+\gamma_{\mathrm{T}}^{-} .
\end{aligned}
$$


The three inequalities $\left(\Lambda_{\mathrm{N}}, \Lambda_{\mathrm{TR}}, \xi_{\mathrm{TL}}\right)^{\top} \succeq 0$ in (28) define together with (27) two independent conditions under which this type of impact can exist, i.e.

$$
\begin{aligned}
& \alpha+\mu \delta>0, \\
& \frac{\gamma_{\mathrm{T}}^{-}}{\gamma_{\mathrm{N}}^{-}} \geq \frac{\delta+\mu \beta}{\alpha+\mu \delta} \cdot \frac{1+\varepsilon_{\mathrm{N}}}{1+\varepsilon_{\mathrm{T}}} .
\end{aligned}
$$

Note in particular that the tangential coefficient of restitution $\varepsilon_{\mathrm{T}}$ only appears in condition (34) and that $\gamma_{\mathrm{N}}^{-}<0$ has been presupposed.

- Stick: This case is defined by a tangential impulse in the interior of the friction interval $\left(\left|\Lambda_{T}\right|<\mu \Lambda_{N}\right)$.

Assume that $\Lambda_{\mathrm{N}}>0$ and $\left|\Lambda_{\mathrm{T}}\right|<\mu \Lambda_{\mathrm{N}}$. It follows from (26) that $\Lambda_{\mathrm{TR}}>0$ and $\Lambda_{\mathrm{TL}}>0$, thus $\xi_{\mathrm{TR}}=0, \xi_{\mathrm{TL}}=0$ and $\xi_{\mathrm{N}}=0$ by (28). Solving now (27) for this case yields $\Lambda_{\mathrm{N}}, \Lambda_{\mathrm{TR}}, \Lambda_{\mathrm{TL}}$, from which one calculates $\Lambda_{\mathrm{T}}$ by (26). Because $\xi_{\mathrm{N}}=\xi_{\mathrm{T}}=0$, the post-impact velocities $\gamma_{\mathrm{N}}^{+}$and $\gamma_{\mathrm{T}}^{+}$are immediately available by (24). One obtains

$$
\begin{aligned}
& \Lambda_{\mathrm{N}}=-\beta \frac{1+\varepsilon_{\mathrm{N}}}{\alpha \beta-\delta^{2}} \gamma_{\mathrm{N}}^{-}+\delta \frac{1+\varepsilon_{\mathrm{T}}}{\alpha \beta-\delta^{2}} \gamma_{\mathrm{T}}^{-}, \\
& \Lambda_{\mathrm{T}}=\delta \frac{1+\varepsilon_{\mathrm{N}}}{\alpha \beta-\delta^{2}} \gamma_{\mathrm{N}}^{-}-\alpha \frac{1+\varepsilon_{\mathrm{T}}}{\alpha \beta-\delta^{2}} \gamma_{\mathrm{T}}^{-}, \\
& \gamma_{\mathrm{N}}^{+}=-\varepsilon_{\mathrm{N}} \gamma_{\mathrm{N}}^{-}, \\
& \gamma_{\mathrm{T}}^{+}=-\varepsilon_{\mathrm{T}} \gamma_{\mathrm{T}}^{-} .
\end{aligned}
$$

We see that both relative velocities, $\gamma_{\mathrm{N}}$ and $\gamma_{\mathrm{T}}$ are are inverted. We call this type of impact "stick" because $\xi_{\mathrm{T}}=0$. Again, the inequalities (28) on $\Lambda_{\mathrm{N}}, \Lambda_{\mathrm{TR}}$ and $\Lambda_{\mathrm{TL}}$ define together with (27) conditions under which this type of impact occurs,

$$
\begin{gathered}
\delta \frac{\gamma_{\mathrm{T}}^{-}}{\gamma_{\mathrm{N}}^{-}}<\beta \frac{1+\varepsilon_{\mathrm{N}}}{1+\varepsilon_{\mathrm{T}}}, \\
(\alpha-\mu \delta) \frac{\gamma_{\mathrm{T}}^{-}}{\gamma_{\mathrm{N}}^{-}}>(\delta-\mu \beta) \frac{1+\varepsilon_{\mathrm{N}}}{1+\varepsilon_{\mathrm{T}}}, \\
(\alpha+\mu \delta) \frac{\gamma_{\mathrm{T}}^{-}}{\gamma_{\mathrm{N}}^{-}}<(\delta+\mu \beta) \frac{1+\varepsilon_{\mathrm{N}}}{1+\varepsilon_{\mathrm{T}}} .
\end{gathered}
$$

Note the exclusively strict inequalities in (39)-(41) $(\langle$ or $\rangle)$ as a consequence on the strict inequality assumption $\left|\Lambda_{T}\right|<\mu \Lambda_{\mathrm{N}}$. Note further that condition (39) follows directly from (41) and (40). Another interesting observation is that the coefficient of friction $\mu$ appears only in the conditions of existence (40) and (41) of this type of impact but neither in the post-impact velocities (37) and (38) $n o r$ in the impulsive forces (35) and (36).

- Forward slip: This case is defined by a tangential impulse at the negative bound of the friction interval $\left(\Lambda_{\mathrm{T}}=-\mu \Lambda_{\mathrm{N}}\right)$.

This case, for which $\xi_{\mathrm{N}}=0, \Lambda_{\mathrm{TR}}=0$ and $\xi_{\mathrm{TL}}=0$, is symmetric to backward slip $\left(\Lambda_{\mathrm{T}}=\mu \Lambda_{\mathrm{N}}\right)$. By using the transformation $\left(\gamma_{\mathrm{T}}^{-}, \varphi\right) \rightarrow\left(-\gamma_{\mathrm{T}}^{-}, \pi-\varphi\right)$, both solutions are transformed into one another (see Figure 4). The impulsive impact forces and the post-impact velocities are

$$
\begin{aligned}
& \Lambda_{\mathrm{N}}=\frac{1+\varepsilon_{\mathrm{N}}}{-\alpha+\mu \delta} \gamma_{\mathrm{N}}^{-}, \\
& \Lambda_{\mathrm{T}}=-\mu \Lambda_{\mathrm{N}},
\end{aligned}
$$




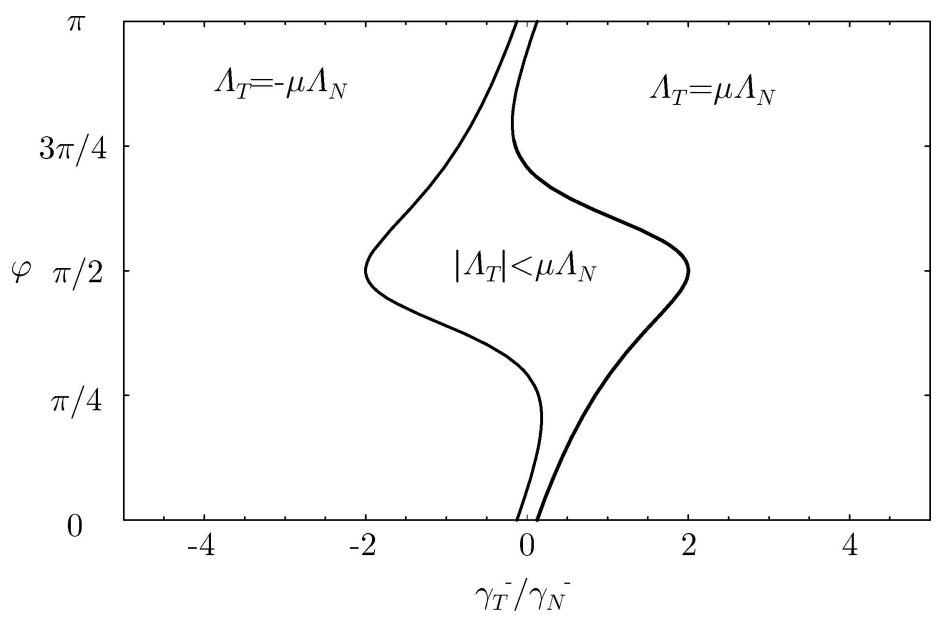

Figure 4. Impact states as function of the pre-impact velocity ratio $\gamma_{\mathrm{T}}^{-} / \gamma_{\mathrm{N}}^{-}$and the impact angle $\varphi$ for sub-critical friction $\left(\varepsilon_{\mathrm{N}}=1, \varepsilon_{\mathrm{T}}=1, \mu=1 / 2\right)$.

$$
\begin{aligned}
& \gamma_{\mathrm{N}}^{+}=-\varepsilon_{\mathrm{N}} \gamma_{\mathrm{N}}^{-}, \\
& \gamma_{\mathrm{T}}^{+}=(\delta-\mu \beta) \Lambda_{\mathrm{N}}+\gamma_{\mathrm{T}}^{-},
\end{aligned}
$$

and the conditions for this type of impact to exist

$$
\begin{aligned}
\alpha-\mu \delta & >0, \\
\frac{\gamma_{\mathrm{T}}^{-}}{\gamma_{\mathrm{N}}^{-}} & \leq \frac{\delta-\mu \beta}{\alpha-\mu \delta} \cdot \frac{1+\varepsilon_{\mathrm{N}}}{1+\varepsilon_{\mathrm{T}}} .
\end{aligned}
$$

For completeness, we also discuss the fourth case to show that it is of no interest for the classification of the impact because of a vanishing normal impulse $\Lambda_{\mathrm{N}}$. Assume, together with $\xi_{\mathrm{N}}=0$, strict positiveness of $\xi_{\mathrm{TR}}$ and $\xi_{\mathrm{TL}}$. This yields by the complementarity conditions (28) the fourth possible case of zerovariables, i.e. $\Lambda_{\mathrm{TR}}=\Lambda_{\mathrm{TL}}=0$. Consequently, the last equation in (27) becomes $0=2 \mu \Lambda_{\mathrm{N}}$, hence $\Lambda_{\mathrm{N}}=0$.

Conditions (33) and (34), (39)-(41), (46) and (47) obtained earlier divide the $\left(\gamma_{\mathrm{T}}^{-} / \gamma_{\mathrm{N}}^{-}, \varphi\right)$ plane into three regions corresponding to the three different types of impacts. Figure 4 shows these domains for a $\operatorname{bar}\left(\Theta_{\mathrm{S}}=1 / 3 m s^{2}\right)$ with a sufficiently small coefficient of friction $\mu$ such that conditions (33) and (46) hold for all angles $\varphi$, i.e. $\mu|\delta|<\alpha$. One clearly sees that for every impact angle $\varphi$ all three types of impact are accessible, depending on the values of the pre-impact velocity ratio. Spheres, for example, are characterized by the unique impact angle $\varphi=\pi / 2=$ constant, which leads in addition to $\delta=0$. Note the symmetry of the picture with respect to point $\left(\gamma_{\mathrm{T}}^{-} / \gamma_{\mathrm{N}}^{-}=0, \varphi=\pi / 2\right)$, which is due to $\alpha(\varphi)=\alpha(\pi-\varphi), \beta(\varphi)=\beta(\pi-\varphi)$ and $\delta(\varphi)=-\delta(\pi-\varphi)$.

As long as $\mu|\delta|<\alpha$, any of the three different impact types exist for any impact angle $\varphi$, as already shown in Figure 4. If this condition is violated due to heavy friction, either of the inequalities (33) or (46) fails. In addition, the inequality sign of either (40) or (41) will change direction when they are divided by $(\alpha-\mu \delta)$ and $(\alpha+\mu \delta)$. For those cases, either backward slip or forward slip can not be reached and stick is valid, instead. Figure 5 depicts the partition of the $\left(\gamma_{\mathrm{T}}^{-} / \gamma_{\mathrm{N}}^{-}, \varphi\right)$ plane for such a supercritical coefficient of friction, in this case $\mu=5 / 3$. The horizontal lines in Figure 5 indicate the impact angles $\varphi$ for which $\mu|\delta|=\alpha$, i.e. the cases for which conditions (34) and (47) have singularities, respectively. Note that the stick domain is unbounded in one direction when the impact angles $\varphi$ are 


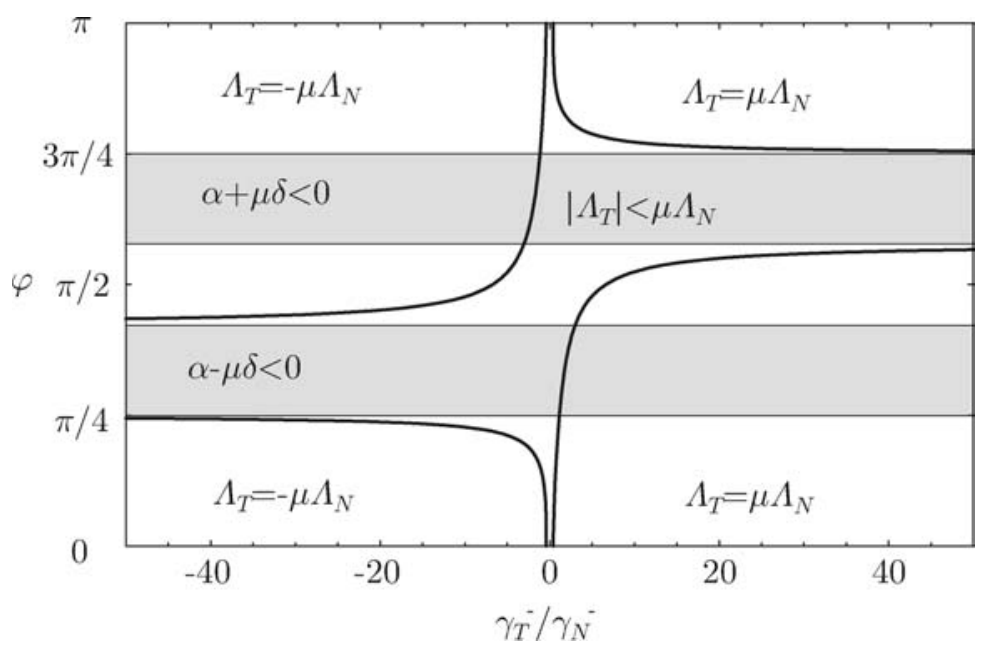

Figure 5. Impact states as function of the pre-impact velocity ratio $\gamma_{\mathrm{T}}^{-} / \gamma_{\mathrm{N}}^{-}$and the impact angle $\varphi$ for super-critical friction $\left(\varepsilon_{\mathrm{N}}=1, \varepsilon_{\mathrm{T}}=1, \mu=5 / 3\right)$.

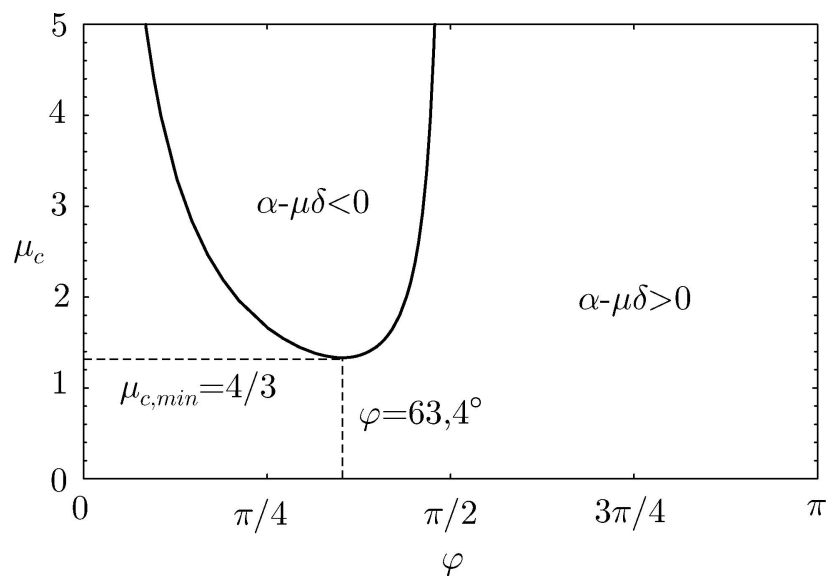

Figure 6. Critical coefficient of friction as function of $\varphi$ for forward $\operatorname{sip}\left(\Lambda_{\mathrm{T}}=-\mu \Lambda_{\mathrm{N}}\right)$.

from the grey shaded area. In this case, either backward slip or forward slip can not occur, independent of the pre-impact velocity ratio $\gamma_{\mathrm{T}}^{-} / \gamma_{\mathrm{N}}^{-}$.

Written as equalities, conditions (33) and (46) provide the critical coefficient of friction $\mu_{\mathrm{c}}$ as a function of the impact angle $\varphi$ for backward or for forward slip, respectively. For the latter case, we obtain $\mu_{\mathrm{c}}=\alpha / \delta$, which is depicted in Figure 6 as a function of the impact angle $\varphi$, see also [4, 17]. The minimum $\mu_{\mathrm{c}, \min }=4 / 3$ is obtained for $\varphi=63.435^{\circ}$. For friction coefficients below this value, the situation depicted in Figure 4 applies and forward slip is possible for all impact angles $\varphi$. If the friction coefficient is increased up to the value $\mu_{\mathrm{c}, \min }$, an impact ending up in forward slip regime at an angle $\varphi=63.435^{\circ}$ requires a pre-impact velocity ratio $\gamma_{\mathrm{T}}^{-} / \gamma_{\mathrm{N}}^{-} \rightarrow-\infty$. For all other angles, a finite pre-impact velocity ratio is still sufficient for forward slip. By a further increase of the friction coefficient, the grey shaded areas in Figure 5 develop from one horizontal line at $\varphi=63.435^{\circ}$. The same behavior is observed in a symmetric manner for backward slip. Some comments on how these grey shaded areas are related to Painlevé problems are made in Section 6. 
In the remaining part of this section we will discuss the phenomenon of sign reversal of the tangential relative velocity at the impact. To do so, Figures 4 and 5 are complemented by the areas for which such a sign reversal occurs. This is conveniently done in terms of the post-impact velocity ratios $\gamma_{\mathrm{T}}^{+} / \gamma_{\mathrm{N}}^{+}$that are obtained for all three types of impact by combining (31) and (32), (37) and (38), and (44) and (45), respectively,

- Backward slip: $\Lambda_{\mathrm{T}}=+\mu \Lambda_{\mathrm{N}}$

$$
\frac{\gamma_{\mathrm{T}}^{+}}{\gamma_{\mathrm{N}}^{+}}=\frac{\delta+\mu \beta}{\alpha+\mu \delta} \cdot \frac{1+\varepsilon_{\mathrm{N}}}{\varepsilon_{\mathrm{N}}}-\frac{1}{\varepsilon_{\mathrm{N}}} \cdot \frac{\gamma_{\mathrm{T}}^{-}}{\gamma_{\mathrm{N}}^{-}},
$$

- Stick: $\left|\Lambda_{T}\right|<\mu \Lambda_{N}$

$$
\frac{\gamma_{\mathrm{T}}^{+}}{\gamma_{\mathrm{N}}^{+}}=\frac{\varepsilon_{\mathrm{T}}}{\varepsilon_{\mathrm{N}}} \cdot \frac{\gamma_{\mathrm{T}}^{-}}{\gamma_{\mathrm{N}}^{-}}
$$

- Forward slip: $\Lambda_{\mathrm{T}}=-\mu \Lambda_{\mathrm{N}}$

$$
\frac{\gamma_{\mathrm{T}}^{+}}{\gamma_{\mathrm{N}}^{+}}=\frac{\delta-\mu \beta}{\alpha-\mu \delta} \cdot \frac{1+\varepsilon_{\mathrm{N}}}{\varepsilon_{\mathrm{N}}}-\frac{1}{\varepsilon_{\mathrm{N}}} \cdot \frac{\gamma_{\mathrm{T}}^{-}}{\gamma_{\mathrm{N}}^{-}} .
$$

By using these equations, we can determine the regions of positive and negative post-impact velocity ratios in the $\left(\gamma_{\mathrm{T}}^{-} / \gamma_{\mathrm{N}}^{-}, \varphi\right)$ plane. Figures 7 and 8 show again the three different impact regimes from Figures 4 and 5, separated from each other by black heavy lines. In addition, the regions of positive $\left(\gamma_{\mathrm{T}}^{+} / \gamma_{\mathrm{N}}^{+}>0\right)$ and negative $\left(\gamma_{\mathrm{T}}^{+} / \gamma_{\mathrm{N}}^{+}<0\right)$ post-impact velocity ratios are marked in white and grey, respectively. Sign reversal of the tangential relative velocity $\left(\operatorname{sgn}\left(\gamma_{\mathrm{T}}^{+}\right)=-\operatorname{sgn}\left(\gamma_{\mathrm{T}}^{-}\right)\right)$is therefore observed for the white areas in the right half $\left(\gamma_{\mathrm{T}}^{-} / \gamma_{\mathrm{N}}^{-}>0\right)$ and for the grey areas in the left half $\left(\gamma_{\mathrm{T}}^{-} / \gamma_{\mathrm{N}}^{-}<0\right)$ of Figures 7 and 8. Remember in this context the sign of the normal relative velocity is always reversed at the impact, which can be seen from Equations (31), (37) and (44). Sign reversal of $\gamma_{\mathrm{T}}$ occurs for the entire stick area due to (49) or (38), and also for some bordering area of the slip domain. The fact that this sign reversal occurs even for slip $\left(\left|\Lambda_{\mathrm{T}}\right|=\mu \Lambda_{\mathrm{N}}\right)$ coincides with the findings of e.g. Walton [8] and Stronge et al. [7] and leads to continuous post-impact velocity ratios $\gamma_{\mathrm{T}}^{+} / \gamma_{\mathrm{N}}^{+}$as function of the pre-impact velocity ratios.

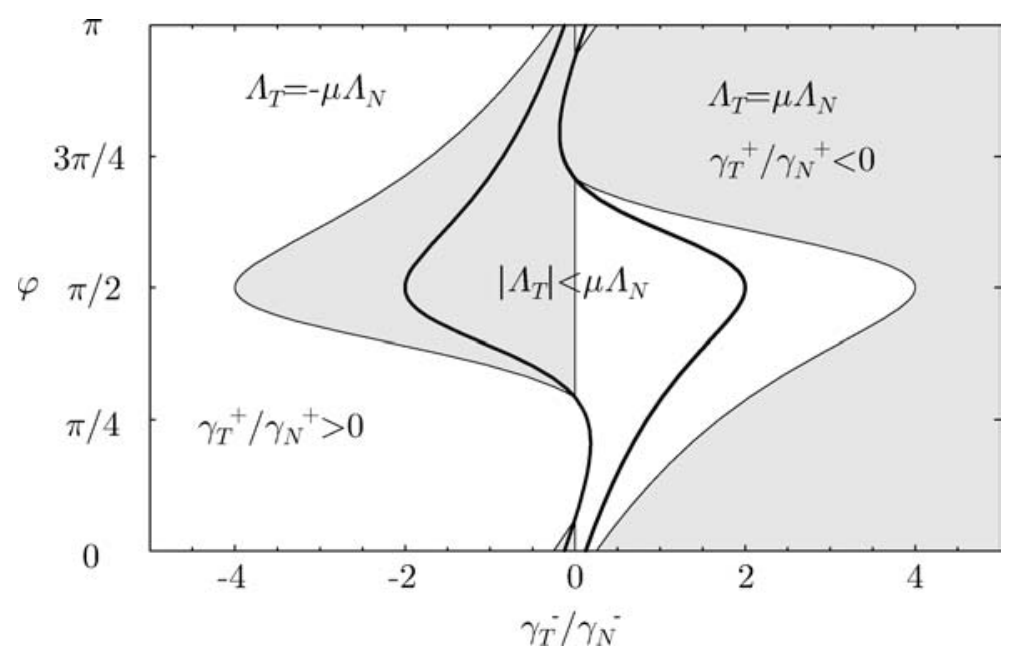

Figure 7. Sign of the post-impact velocity ratio $\gamma_{\mathrm{T}}^{+} / \gamma_{\mathrm{N}}^{+}$as function of the pre-impact velocity ratio ratio $\gamma_{\mathrm{T}}^{-} / \gamma_{\mathrm{N}}^{-}$and the impact angle $\varphi$ for sub-critical friction $\left(\varepsilon_{\mathrm{N}}=1, \varepsilon_{\mathrm{T}}=1, \mu=1 / 2\right)$. 


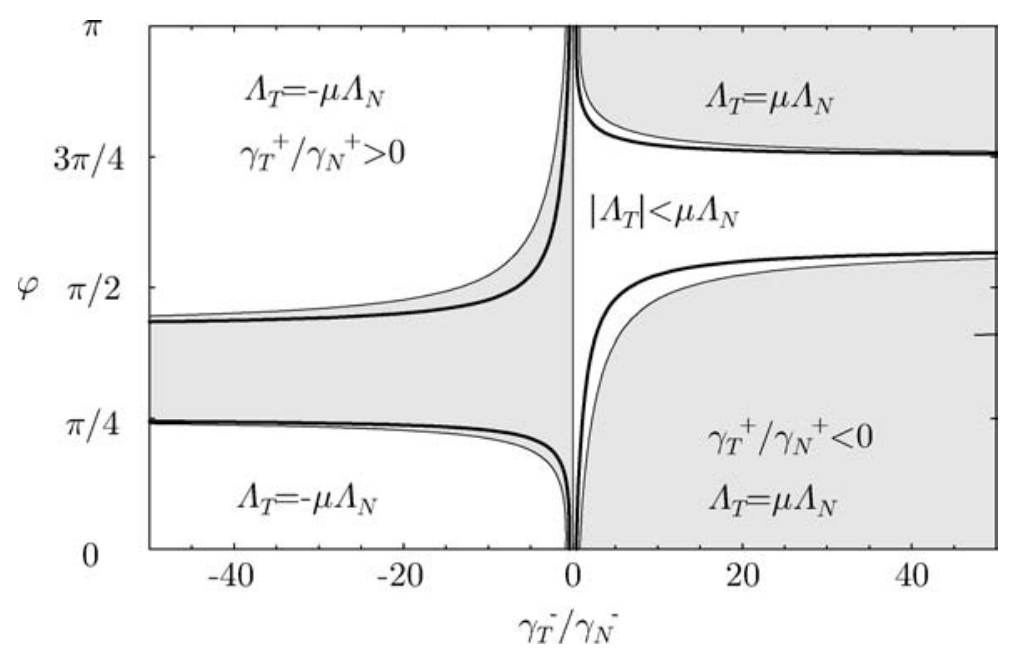

Figure 8. Sign of the post-impact velocity ratio $\gamma_{\mathrm{T}}^{+} / \gamma_{\mathrm{N}}^{+}$as function of the pre-impact velocity ratio ratio $\gamma_{\mathrm{T}}^{-} / \gamma_{\mathrm{N}}^{-}$and the impact angle $\varphi$ for super-critical friction $\left(\varepsilon_{\mathrm{N}}=1, \varepsilon_{\mathrm{T}}=1, \mu=5 / 3\right)$.

In order to avoid possible confusion caused by the reader's intuitive interpretation of the terms "stick" and "slip", we want to point out again that they are used and have to be understood according to their definition given at the beginning of this section. Slip means that $\left|\Lambda_{T}\right|=\mu \Lambda_{N}$, whereas stick means $\left|\Lambda_{\mathrm{T}}\right|<\mu \Lambda_{\mathrm{N}}$, independent of the values of the pre- or post-impact tangential relative velocities $\gamma_{\mathrm{T}}^{-}, \gamma_{\mathrm{T}}^{+}$.

Figure 9 depicts the post-impact velocity ratio as a function of the pre-impact velocity ratio according to Equations (48)-(50) for two different angles, $\varphi=\pi / 2$ and $\varphi=\pi / 4$. As a general result, this function is continuous for any chosen value of $\varphi$. For $\varphi=\pi / 2$, the regions of stick, slip and sign reversal are also depicted. Finally, Figure 10 shows the post-impact velocity ratio for the whole set of feasible angles.

To get a physical idea of the slip-sign reversal, consider an elastic sphere striking sufficiently steep (but not too steep) a plane. Assume that the initial sliding process in the contact region passes into

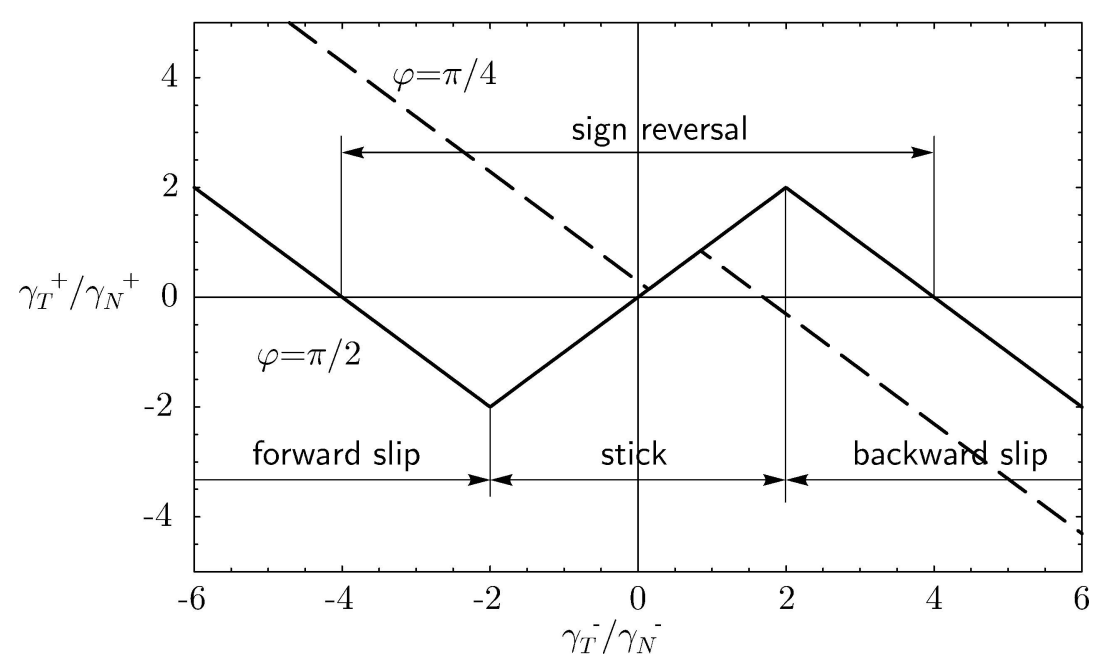

Figure 9. Post-impact velocity ratio $\gamma_{\mathrm{T}}^{+} / \gamma_{\mathrm{N}}^{+}$as function of $\gamma_{\mathrm{T}}^{-} / \gamma_{\mathrm{N}}^{-}$for $\varphi=\pi / 2$ (solid line) and $\varphi=\pi / 4$ (dashed line) with $\varepsilon_{\mathrm{N}}=1, \varepsilon_{\mathrm{T}}=1$ and $\mu=1 / 2$. 


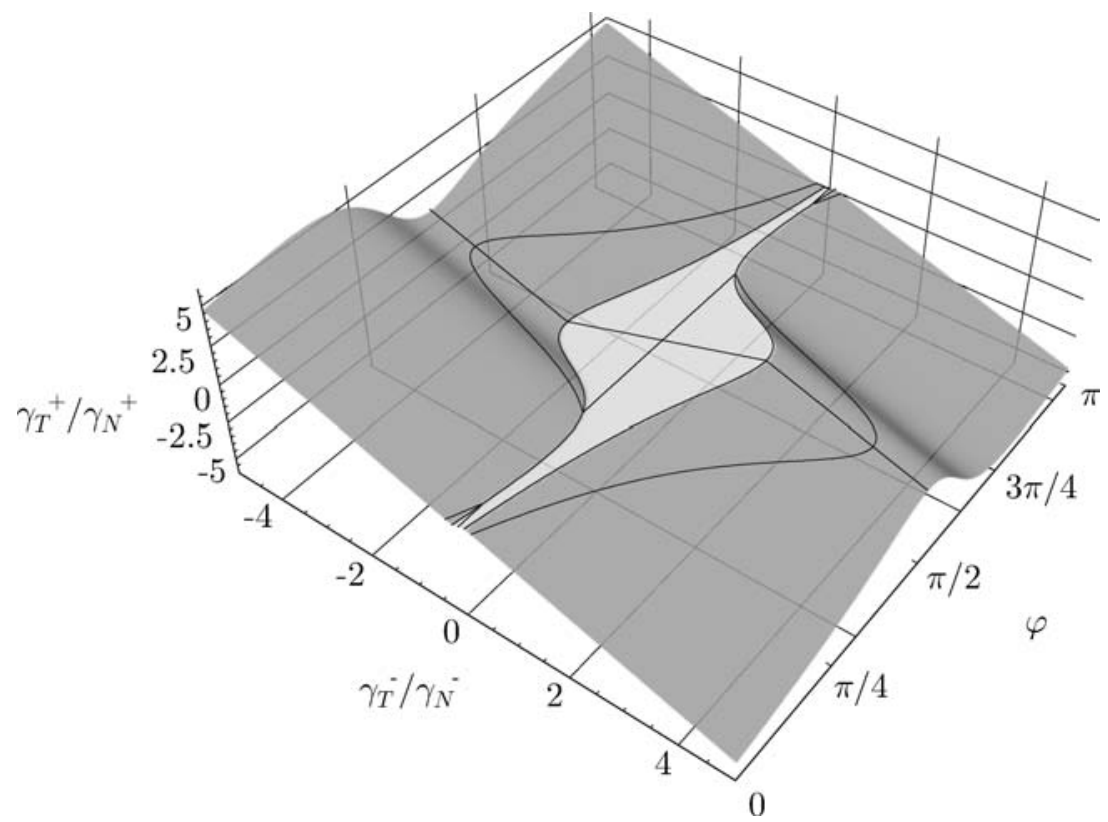

Figure 10. Post-impact velocity ratio $\gamma_{\mathrm{T}}^{+} / \gamma_{\mathrm{N}}^{+}$as function of $\gamma_{\mathrm{T}}^{-} / \gamma_{\mathrm{N}}^{-}$for all feasible angles $\varphi$ with $\varepsilon_{\mathrm{N}}=1, \varepsilon_{\mathrm{T}}=1$ and $\mu=1 / 2$.

sticking. Before separation, the stored tangential strain energy in the contact region causes the relative surface velocity to reverse its direction. At more steeper angles of incidence, the initial sliding phase might disappear and the process will start immediately with tangential loading and unloading but without losses due to sliding friction.

\section{Some Comments on Painlevé Problems}

The value of $\mu_{\mathrm{c}, \min }=4 / 3$ from Figure 6 in the previous section is well known as the minimal friction coefficient necessary for the Painlevé paradox to arise, as first published by Painlevé [10] in 1895 for a similar example. We want to spend at least some comments on this phenomenon because of its extraordinary importance. Painlevé problems are usually attacked by assuming a sliding motion under the influence of Coulomb friction of the system, here the sliding bar. By this assumption, collisions have clearly been excluded from the problem. Therefore, one is tempted to study the motion of the bar classically, i.e. by only taking into account its equations of motion on acceleration level by excluding any kind of impacts, as done in the original contribution [10]. As a consequence, non-uniqueness or even non-existence of solution may occur, see e.g. Génot and Brogliato [11], and Leine et al. [12] for a thorough discussion of this phenomenon.

In the following, we try to explain the role that the frictional impact law plays for the Painleve paradox. We assume the bar to touch the surface very gently by a small pre-impact velocity $\gamma_{\mathrm{N}}^{-}$, which corresponds in the limit to a pre-impact sliding state with $\gamma_{\mathrm{N}}^{-} \uparrow 0$ and $\gamma_{\mathrm{T}}^{-} \neq 0$.

We consider firstly sub-critical friction (Figure 4) and investigate how the frictional impact law acts on the assumed pre-impact sliding state. By the chosen values of $\gamma_{\mathrm{N}}^{-}$and $\gamma_{\mathrm{T}}^{-}$, we end up at the very far right or left in Figure $4, \gamma_{\mathrm{T}}^{-} / \gamma_{\mathrm{N}}^{-} \longrightarrow \pm \infty$, which corresponds for arbitrary angles $\varphi$ to backward and forward slips according to Equations (29)-(32) and (42)-(45), respectively. By setting $\gamma_{\mathrm{N}}^{-}=0$ in these 
equations, one obtains step by step $\Lambda_{\mathrm{N}}=0, \Lambda_{\mathrm{T}}=0, \gamma_{\mathrm{N}}^{+}=0, \gamma_{\mathrm{T}}^{+}=\gamma_{\mathrm{T}}^{-}$. Thus, there is no impulsive force and the tangential as well as the normal relative velocities remain remain unchanged. Loosely speaking, this behavior agrees with the intuitively expected result that nothing discontinuous happens without a collision. An important conclusion from this case is that the impact law provides the right results, even if it is applied in the absence of a collision!

Let us now assume supercritical friction as for angles $\varphi$ from the grey shaded areas in Figure 5. For clarity, we will only discuss the situation associated with the lower grey band in Figure 5. The upper grey band works symmetrically. Suppose first a pre-impact velocity ratio $\gamma_{\mathrm{T}}^{-} / \gamma_{\mathrm{N}}^{-} \longrightarrow+\infty$. In this case, backward slip applies $\left(\Lambda_{\mathrm{T}}=+\mu \Lambda_{\mathrm{N}}\right)$, and the post-impact velocities are obtained in the same manner as for sub-critical friction. As a result, none of the velocities will change and there is no impact. Suppose now that $\gamma_{\mathrm{T}}^{-} / \gamma_{\mathrm{N}}^{-} \longrightarrow-\infty$, for instance, set by initial conditions. We see from Figure 5 that forward slip $\left(\Lambda_{\mathrm{T}}=-\mu \Lambda_{\mathrm{N}}\right)$ does not exist for values of $\varphi$ from the lower grey band. Instead, stick is valid, which can be evaluated through Equations (35)-(38). By setting $\gamma_{\mathrm{N}}^{-}=0$, one obtains from (35) and (36) non-vanishing positive impulsive forces $\Lambda_{\mathrm{N}}$ and $\Lambda_{\mathrm{T}}$, a vanishing normal post-impact velocity $\gamma_{\mathrm{N}}^{+}=0$ from (37), and a tangential post-impact velocity that is inverted according to (38). Thus, the tangential contact velocity $\gamma_{\mathrm{T}}$ changes sign and the normal contact velocity $\gamma_{\mathrm{N}}$ remains zero, even though the impulsive force $\Lambda_{\mathrm{N}}$ is non-zero. This process corresponds to an instantaneous dynamic locking process, called in [1] an impact without collision. A potential contact loss after this blocking event evolves from this post-impact state, but is governed by the classical Newton-Euler equations on acceleration level, see e.g. [17] for some numerical results with $\varepsilon_{\mathrm{N}}=\varepsilon_{\mathrm{T}}=0$. Consequently, the Newton-Euler equations on acceleration level are not sufficient to describe the collision-free dynamics of the system.

\section{Comparison of Different Frictional Impact Models}

In this section, we compare different models of frictional impacts with the non-smooth inequality complementarity method presented so far. We show that Walton's [8] collision operator for spheres is a special case of the non-smooth complementarity method, providing the same results as a consequence. We further show that these results coincide - under certain conditions - with a lumped mass models approach. In particular, we take our results of the impacting bar from Section 5 and compare them with the findings of Stronge et al. [7].

In the first part of this section, we relate the non-smooth inequality complementarity method to Walton's frictional collision operator for two identical spheres impacting against each other. The impact equations for a rigid sphere (midpoint $\mathrm{S}$, radius $s$ ) against an inelastic half-space may easily be derived from the configuration of the bar shown in Figure 3. For an impacting sphere, the radius vector $\mathbf{r}_{\mathrm{SC}}$ from the sphere's midpoint $S$ to the contact point $C$ is always perpendicular to the contact plane, which corresponds to a unique impact angle $\varphi \equiv \pi / 2$. This angle has to be used in (16) and all the subsequent equations together with the sphere's moment of inertia $\Theta_{\mathrm{S}}=2 / 5 \mathrm{~ms}^{2}$. In particular, the functions $(\alpha, \beta, \delta)(\varphi)$ in (22) become constants with values $\alpha=1 / m, \beta=1 / m(1+(5 / 2))$ and $\delta=0$. As a consequence on $\delta=0$, the normal and the tangential momentum Equations (23) are decoupled such that the impacts in the normal and the tangential directions can be solved one after the other. Moreover, the conditions (33) and (46) on backward and forward slips to exist, both simplify to $\alpha>0$, which holds always true. Thus, the impact of a sphere is always sub-critical with respect to the coefficient of friction, and configurations in the $\left(\gamma_{\mathrm{T}}^{-} / \gamma_{\mathrm{N}}^{-}, \varphi\right)$ plane of the type depicted in Figure 5 as well as Painlevé problems can not occur. By introducing the quantity $K:=\Theta_{\mathrm{S}} /\left(m s^{2}\right)=2 / 5$, the remaining conditions (34), (40), (41), and (47) for an impact type to occur simplify to 
- Backward slip, if

$$
\frac{\gamma_{\mathrm{T}}^{-}}{\gamma_{\mathrm{N}}^{-}} \geq \mu\left(1+\frac{1}{K}\right) \cdot \frac{1+\varepsilon_{\mathrm{N}}}{1+\varepsilon_{\mathrm{T}}},
$$

- Stick, if $\left|\Lambda_{\mathrm{T}}\right|<\mu \Lambda_{\mathrm{N}}$

$$
\left|\frac{\gamma_{\mathrm{T}}^{-}}{\gamma_{\mathrm{N}}^{-}}\right|<\mu\left(1+\frac{1}{K}\right) \cdot \frac{1+\varepsilon_{\mathrm{N}}}{1+\varepsilon_{\mathrm{T}}},
$$

- Forward slip, if

$$
\frac{\gamma_{\mathrm{T}}^{-}}{\gamma_{\mathrm{N}}^{-}} \leq \mu\left(1+\frac{1}{K}\right) \cdot \frac{1+\varepsilon_{\mathrm{N}}}{1+\varepsilon_{\mathrm{T}}} .
$$

Finally, the post-impact velocity ratios (48)-(50) can be written as

- Backward slip:

$$
\frac{\gamma_{\mathrm{T}}^{+}}{\gamma_{\mathrm{N}}^{+}}=\mu\left(1+\frac{1}{K}\right)\left(1+\frac{1}{\varepsilon_{\mathrm{N}}}\right)-\frac{1}{\varepsilon_{\mathrm{N}}} \cdot \frac{\gamma_{\mathrm{T}}^{-}}{\gamma_{\mathrm{N}}^{-}},
$$

- Stick:

$$
\frac{\gamma_{\mathrm{T}}^{+}}{\gamma_{\mathrm{N}}^{+}}=\frac{\varepsilon_{\mathrm{T}}}{\varepsilon_{\mathrm{N}}} \cdot \frac{\gamma_{\mathrm{T}}^{-}}{\gamma_{\mathrm{N}}^{-}}
$$

- Forward slip:

$$
\frac{\gamma_{\mathrm{T}}^{+}}{\gamma_{\mathrm{N}}^{+}}=-\mu\left(1+\frac{1}{K}\right)\left(1+\frac{1}{\varepsilon_{\mathrm{N}}}\right)-\frac{1}{\varepsilon_{\mathrm{N}}} \cdot \frac{\gamma_{\mathrm{T}}^{-}}{\gamma_{\mathrm{N}}^{-}} .
$$

Together with the impact law in the normal direction (31), (37), and (44),

$$
\gamma_{\mathrm{N}}^{+}=-\varepsilon_{\mathrm{N}} \gamma_{\mathrm{N}}^{-}
$$

Equations (51)-(56) provide the full collision operator that is necessary to evaluate the frictional impact of a sphere against a rigid half-space. In order to determine the post-impact relative velocities $\gamma_{\mathrm{N}}^{+}$and $\gamma_{\mathrm{T}}^{+}$, one has to proceed as follows: From the known pre-impact velocities $\gamma_{\mathrm{N}}^{-}$and $\gamma_{\mathrm{T}}^{-}$, one first checks by (51)-(53) which of the impact states apply. In a second step, the associated post-impact velocity ratio is determined according to (54)-(56), which together with (57) gives the values for $\gamma_{\mathrm{N}}^{+}$and $\gamma_{\mathrm{T}}^{+}$.

Equations (54)-(56) are found precisely in this form in Walton [8], where he applies instantaneous collision operators to the impacts between identical spheres to simulate particle-loaded flows. The advantage of this approach compared with a compliance-based model of the frictional contacts is the fast numerical evaluation because a resolution of the high-frequency dynamics during the impact can be avoided. The valid impact state is determined in [8] by an intuitive method, assuming first slip and then correcting to stick if necessary, but is identical to (51)-(53). Contrary to our frictional impact of a bar against a rigid half-space, Walton considers two identical spheres striking each other. However, this incident can be decomposed into two symmetric impacts of spheres against a rigid impact plane. Consequently, Walton's collision operator is a special case of and therefore fully included in the nonsmooth inequality complementarity approach. 


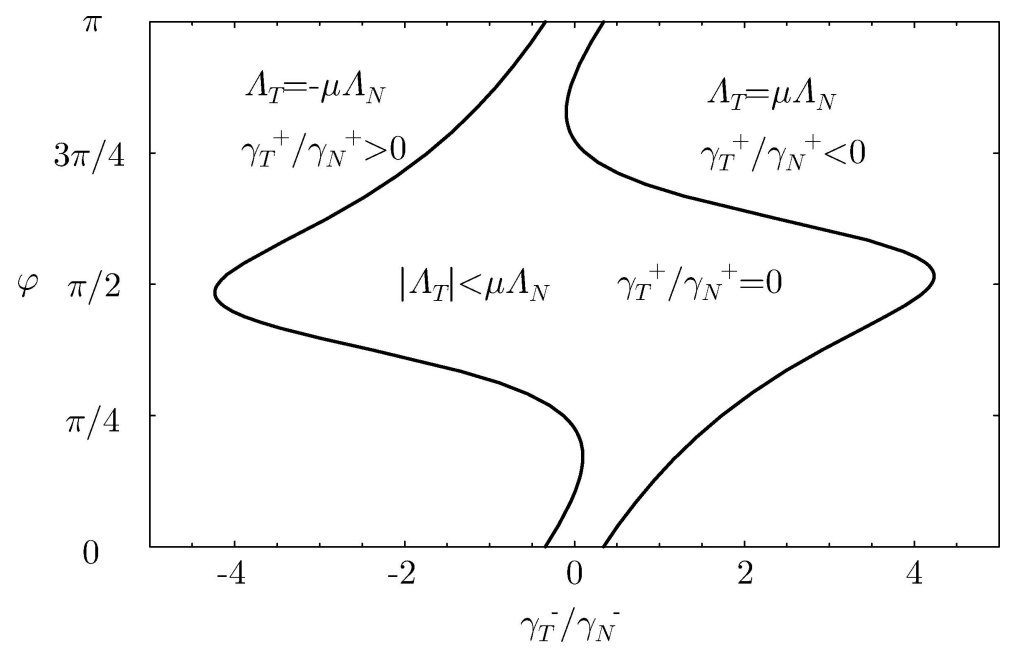

Figure 11. Impact solutions and sign of the post-impact velocity ratio for a bar with the moment of inertia of a sphere $K=2 / 5$ as function of $\gamma_{\mathrm{T}}^{-} / \gamma_{\mathrm{N}}^{-}$and $\varphi$ with $\varepsilon_{\mathrm{N}}=1, \varepsilon_{\mathrm{T}}=0$ and $\mu=3 / 5$.

To give at least one illustrative example on how the impact of spheres is related to the impact of bars, we have evaluated conditions (33) and (34), (39)-(41), (46) and (47) for a bar with a non-uniform density such that the moment of inertia equals the one of a sphere, $\Theta_{\mathrm{S}}=2 / 5 \mathrm{~ms}^{2}$. The resulting partitioning of the $\left(\gamma_{\mathrm{T}}^{-} / \gamma_{\mathrm{N}}^{-}, \varphi\right)$ plane into the three different impact regimes is depicted in Figure 11, where the normal and tangential coefficients of restitution have been chosen as $\varepsilon_{\mathrm{N}}=1, \varepsilon_{\mathrm{T}}=0$, and the coefficient of friction as $\mu=3 / 5$. For $\varphi=\pi / 2$, one obtains the impact behavior of a sphere with the values of $\gamma_{\mathrm{T}}^{-} / \gamma_{\mathrm{N}}^{-}$that separate the three different impact regimes according to (51)-(53). As a consequence on $\varepsilon_{\mathrm{T}}=0$, no sign reversal can occur for this case, which is easily seen from Equations (54)-(56).

In the remaining part of this section, we compare the non-smooth inequality complementarity approach with the lumped mass model from Stronge et al. [7]. In this work, the authors propose an analytical approach to the frictional planar single impact of two bodies of arbitrary shape against each other. They assume that the colliding bodies undergo only small deformations in a small region localized around the initial point of contact, causing large contact forces that drive the bodies apart during the impact. The rigid body coordinates, in our notation $\mathbf{q}$, are assumed to be constant during the collision, which leads to constant inertia properties as well.

In contrast, the authors allow for local contact displacements $u_{i}$ as depicted in Figure 12. In their lumped-parameter model, the compliance of the small deformable region around the contact point is represented by discrete massless elements, which are oriented in normal and tangential direction. The contact dynamics equations are formulated in local contact variables according to (23) but with the velocity differences and impulsive forces having been replaced by relative accelerations $\left(\dot{\gamma}_{\mathrm{N}}, \dot{\gamma}_{\mathrm{T}}\right)$ and finite forces $\left(\dot{\Lambda}_{\mathrm{N}}, \dot{\Lambda}_{\mathrm{T}}\right)$. The impact process itself is classically decomposed into a phase of compression and a subsequent period of restitution. Compression terminates with zero normal relative velocity, and restitution with zero normal force, respectively. Contact compliance in normal direction is taken into account by a piecewise linear spring with hysteresis, from which the weaker branch acts during compression and the stiffer during restitution. This behavior of the compliant elements is depicted in the right diagram of Figure 12. Stronge's [7] energetic coefficient of restitution $e_{*}$ is defined by the square root of the associated stiffness ratio. In tangential direction, compliance and friction are modelled by a linear spring and a Coulomb friction element in series. 

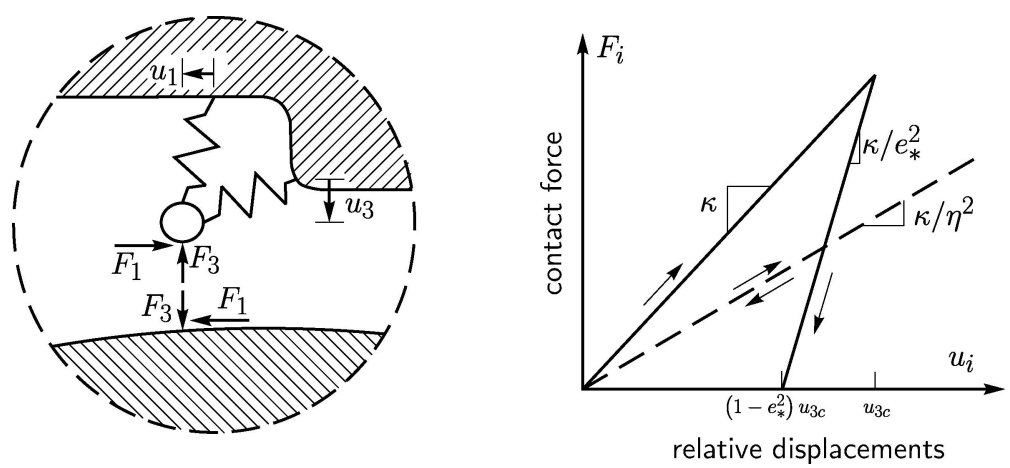

Figure 12. Stronge's [7] analytical model of the contact between two hard bodies with separate normal and tangential compliance at the contact point.

This impact model results in a set of piecewise linear second-order ordinary differential equations solved analytically for the different phases of the impact, and afterwards pasted together. By the nature of this approach, a higher order dependency of the post-impact velocity ratio $\gamma_{\mathrm{T}}^{+} / \gamma_{\mathrm{N}}^{+}$on the pre-impact velocity ratio $\gamma_{\mathrm{T}}^{-} / \gamma_{\mathrm{N}}^{-}$is observed. However, for "gross slip", which is slip without sign reversal or stick within the impact process, the following result is reported:

$$
\frac{v_{1}\left(t_{f}\right)}{v_{3}\left(t_{f}\right)}=\frac{\delta+\mu \operatorname{sgn}\left(v_{1}(0)\right) \beta}{\alpha+\mu \operatorname{sgn}\left(v_{1}(0)\right) \delta} \cdot \frac{1+e_{*}}{e_{*}}-\frac{1}{e_{*}} \cdot \frac{v_{1}(0)}{v_{3}(0)} .
$$

Expressed in our notation with $v_{1}(0)=\gamma_{\mathrm{T}}^{-}, v_{1}\left(t_{f}\right)=\gamma_{\mathrm{T}}^{+}$for the tangential terms and $v_{3}(0)=$ $\gamma_{N}^{-}, v_{3}\left(t_{f}\right)=\gamma_{\mathrm{N}}^{+}$for the normal terms, one obtains precisely the post-impact velocity ratio for backward and forward slip form (48) and (50). Hence, Stronge's energetic coefficient of restitution $e_{*}$ is equivalent to the kinematic coefficient of restitution $\varepsilon_{\mathrm{N}}$ in the case of gross slip. This result is a direct consequence on the linearity of the equations of motion used in [7] together with the constant ratio of the tangential and the normal force in the case of sliding, i.e. $\int_{\Delta t}\left(\dot{\Lambda}_{\mathrm{T}}(t) / \dot{\Lambda}_{\mathrm{N}}(t)\right) d t=\int_{\Delta t} \mu d t=\mu \Delta t=\Lambda_{\mathrm{T}} / \Lambda_{\mathrm{N}} \Delta t$ when $\Delta t$ is the impact duration.

Finally, we want to show by at least one example the difference between Stronge's approach and the non-smooth inequality complementarity method when gross slip does not apply everywhere. In Figure 13, which has been taken from [7], the impact of a sphere against a rough half-space is evaluated. The tangential post-impact relative velocity $\gamma_{\mathrm{T}}^{+}=v_{1}\left(t_{f}\right)$ is displayed as a function of the tangential pre-impact velocity $\gamma_{\mathrm{T}}^{-}=v_{1}(0)$, both normalized by the normal pre-impact velocity $\gamma_{\mathrm{N}}^{-}=v_{3}(0)$. Solid lines are due to the model of Stronge, whereas dashed lines result from Walton's collision operator. The figure can be divided into two parts with respect to Walton's results: The part of the curves above the light dashed horizontal line correspond to gross slip and agree fully with Stronge's results, given by the formula

$$
-\frac{v_{1}\left(t_{f}\right)}{v_{3}(0)}=\frac{\delta+\mu \operatorname{sgn}\left(v_{1}(0)\right) \beta}{\alpha+\mu \operatorname{sgn}\left(v_{1}(0)\right) \delta}\left(1+e_{*}\right)-\frac{v_{1}(0)}{v_{3}(0)} .
$$

This equation is obtained from (58) by setting $v_{3}\left(t_{f}\right)=-e_{*} v_{3}(0)$, which is shown in [7] to hold for gross slip, and which is also recognized as the normal Newtonian impact law (31), (37) and (44). For the area below the light dashed line, both approaches yield different results. Experiments of Foerester 


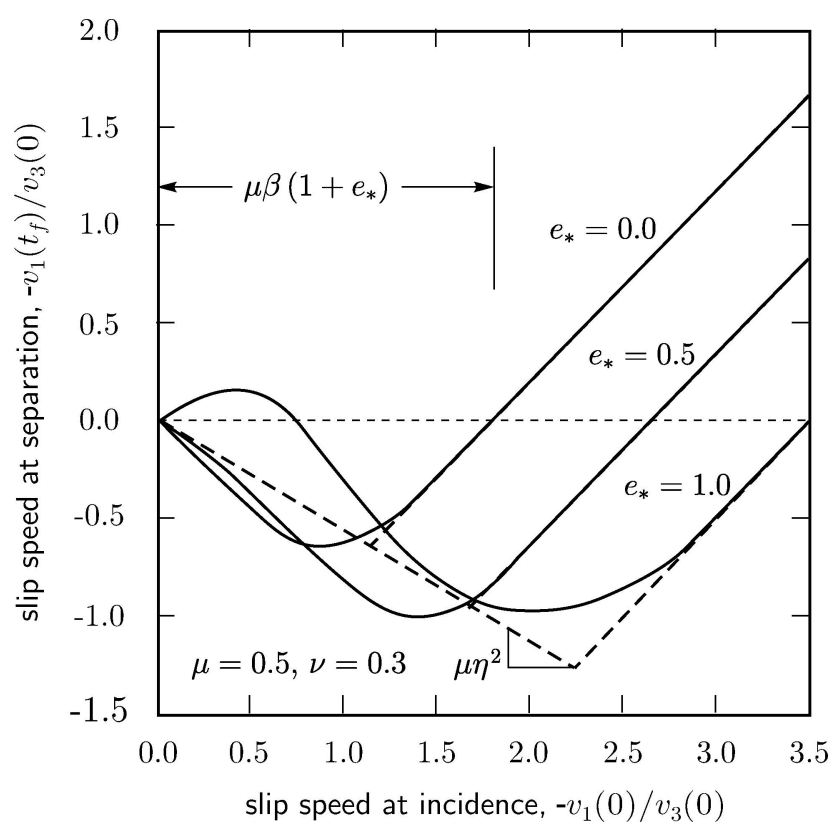

Figure 13. Terminal slip speed $v_{1}\left(t_{f}\right)$ at contact point $\mathrm{C}$ as function of incident slip speed $v_{1}(0)$, both normalized by the incident normal contact velocity $v_{3}(0)$, for an impact of a sphere against a rough, inelastic half-space. Dashed lines are obtained from Walton [8], solid lines by Stronge et al. [7]. The figure is taken from [7].

et al. [18] with small spheres show, however, that both Stronge's lumped mass model approach as well as Walton's collision operator and, thus, the non-smooth inequality complementarity method lie within the same margin of error to the measurements. To obtain Walton's result in Figure 13 by the non-smooth inequality complementarity method, the following parameters have to be chosen: A friction coefficient $\mu=0.5$ as also used by Stronge (see Figure 13), normal restitution coefficients $\varepsilon_{\mathrm{N}} \in\{0,0.5,1\}$ that equal in the case of gross slip Stronge's energetic coefficient $e_{*}$, and a tangential restitution coefficient that is called in [7] the coefficient for stick $\mu \eta^{2}=\varepsilon_{\mathrm{T}}=0.605$.

One advantage of Stronge's model is that it allows for an analytical solution of the collision process because the underlying differential equations are linear for each mode of the impact. However, as soon as spatial friction is considered, one has to expect non-linearities arising from the direction of the friction force. Similar problems occur when a Hertz contact is modelled. For those cases, analytical solutions can hardly be expected. Nevertheless, it would still be possible to evaluate the impact behavior numerically. This would require a numerical code that is capable of treating discontinuities, which are caused by the friction law and the hysteresis behavior in the normal direction. Such a code would be a code from nonsmooth mechanics, because the particular constitutive equations used by Stronge are standard elements from this field. Stronge's model - as well as many other approaches - does not provide an impact law in the sense of constitutive equations but has already to be regarded as a mechanical system, which can be treated numerically by methods from non-smooth dynamics. In general, it does not make much sense to implement in a numerical software package such advanced models as the basis for impact situations. More useful are basic elements from which such systems can be designed. One among them is the frictional Newtonian impact law in inequality form, as presented and applied in this paper. Based on a concise and consistent mathematical setting, it is excellently suited for numerical implementation and provides at the same time a sound basis for advanced models. 


\section{Conclusions}

In this paper, we have presented the full non-smooth inequality complementarity method for planar frictional impact-contacts of Newton-Coulomb type based on a formulation using the set-valued maps Upr and Sgn. Each contact has been characterized by three parameters, i.e. the friction coefficient and the normal and tangential restitution coefficients.

The method has been applied to the example of a rigid bar striking an inelastic half-space. The resulting inequality problem has been formulated in contact coordinates as a linear complementarity problem in standard form and evaluated to derive the conditions under which the three different impact states "backward slip", "stick", and "forward slip" exist. It has been shown that each of these states is accessible by certain values of the pre-impact velocity ratio for sub-critical friction, independent of the impact angle of the bar. For super-critical friction and steep impact angles, only one out of the two slip states continues to exist. This phenomenon is related to the Painlevé paradox, which has also been addressed by showing the existence of impacts without collisions. As a general result, the impact law yields for any feasible pre-impact velocity a unique post-impact velocity, which can be seen in Figures 4 and 5, from the proper partitioning of the $\left(\gamma_{\mathrm{T}}^{-} / \gamma_{\mathrm{N}}^{-}, \varphi\right)$ plane into the three impact regimes. Sign reversals of the tangential relative velocity during the impact and their occurrences in connection with the tangential impact coefficient have also been discussed (Figures 7 and 8) and are even met for slip. Another observation made is that the non-smooth inequality complementarity method leads to post-impact velocity ratios $\gamma_{\mathrm{T}}^{+} / \gamma_{\mathrm{N}}^{+}$, which are continuous functions of the pre-impact velocity ratios $\gamma_{\mathrm{T}}^{-} / \gamma_{\mathrm{N}}^{-}$and of the impact angle $\varphi$, best seen from Figure 10.

As a special case, the collision of a rigid sphere against an inelastic half-space has been derived from the impact configuration of the rigid bar. The full collision operator for this event has been presented by showing equivalence with the model of Walton. The non-smooth inequality approach has further been compared with a lumped mass model using Stronge's energetic coefficient of restitution. For a rigid bar striking an inelastic half-space, the lumped mass model and the non-smooth inequality approach yield the same results when gross slip applies. In this particular case, the energetic and the kinematic coefficients of restitution are identical. For spheres, all three approaches coincide for gross slip, whereas for steeper angles of incidence the results of the lumped mass model deviate from the other two approaches.

\section{Acknowledgements}

This work was conducted within the SICONOS project being part of the IST Programme of the European Community. Financial support was received from the Swiss Federal Office for Education and Science.

\section{References}

1. Moreau, J. J., 'Unilateral contact and dry friction in finite freedom dynamics', in Non-Smooth Mechanics and Applications, J. J. Moreau and P. D. Panagiotopoulos (eds.), CISM Courses and Lectures, Vol. 302, Springer Verlag, Wien, 1988 , pp. 1-82.

2. Glocker, Ch., Set-Valued Force Laws - Dynamics of Non-Smooth Systems, Lecture Notes in Applied Mechanics, Vol. 1, Springer Verlag, Berlin, Heidelberg, 2001.

3. Moreau, J. J., 'Bounded variation in time', in Topics in Nonsmooth Mechanics, J. J. Moreau, P. D. Panagiotopoulos, and G. Strang (eds.), Birkhäuser Verlag, Basel, 1988, pp. 1-74.

4. Pfeiffer, F. and Glocker, Ch., Multibody Dynamics with Unilateral Contacts, Wiley, New York, 1996. 
5. Brogliato, B., Nonsmooth Impact Mechanics. Models, Dynamics and Control, Lecture Notes in Control and Information Sciences, Vol. 220, Springer Verlag, Heidelberg, New York, 1996.

6. Brach, R. M., Mechanical Impact Dynamics, Wiley, New York, 1991.

7. Stronge, W. J., James, R., and Ravani, B., 'Oblique impact with friction and tangential compliance', Philosophical Transactions of the Royal Society of London A 359, 2001, 2447-2465.

8. Walton, O, R., 'Numerical simulation of inelastic, frictional particle-particle interactions', in Particulate Two-Phase Flow, M. C. Roco (ed.), Butterworth-Heinemann, New York, 1992, pp. 884-911.

9. Wang, Y. and Mason, M. T., 'Modeling impact dynamics for robotic operations', in Proceedings of the 1987 IEEE International Conference on Robotics and Automation, Raleigh, NC, 1987, pp. 678-685.

10. Painlevé, P., 'Sur les lois du frottement de glissement', Comptes Rendu des Séances de l'Academie des Sciences 121, 1895, $112-115$.

11. Génot, F. and Brogliato, B., 'New results on Painlevé paradoxes', European Journal of Mechanics A/Solids 18, 1999, 653-677.

12. Leine, R. I., Brogliato, B., and Nijmeijer H., 'Periodic motion and bifurcations induced by the Painlevé paradox', European Journal of Mechanics A/Solids 21, 2002, 869-896.

13. Cottle, R. W., Pang, J. S., and Stone, R. E., The Linear Complementarity Problem, Computer Science and Scientific Computing, Academic Press, London, 1992.

14. Murty, K. G., Linear Complementarity, Linear and Nonlinear Programming, Sigma Series in Applied Mathematics, Vol. 3 , Heldermann Verlag, Berlin, 1988.

15. Glocker, Ch. and Studer, C., 'Formulation and preparation for numerical evaluation of linear complementarity systems in dynamics', Multibody System Dynamics, in press.

16. Moreau, J. J., 'Some numerical methods in multibody dynamics: Application to granular materials', European Journal of Mechanics A/Solids 21(Suppl. 4), 1994, 93-114.

17. Glocker, Ch., Dynamik von Starrkörpersystemen mit Reibung und Stössen, VDI-Fortschrittberichte Mechanik/ Bruchmechanik, Reihe 18, Nr. 182, VDI-Verlag, Düsseldorf, 1995.

18. Foerester, S. F., Louge, M. Y., Chang, H., and Allia, K., 'Measurement of collision properties of small spheres', Physics of Fluids 6, 1994, 1108-1113. 